\title{
The Chloroflexi supergroup is metabolically diverse and representatives have novel genes for non-photosynthesis based $\mathrm{CO}_{2}$ fixation
}

Jacob A. West-Roberts, Paula B. Matheus-Carnevali, Marie Charlotte Schoelmerich, Basem Al-Shayeb, Alex D. Thomas, Allison Sharrar, Christine He, Lin-Xing Chen, Adi Lavy, Ray Keren, Yuki Amano, Jillian F. Banfield

\begin{abstract}
The Chloroflexi superphylum have been investigated primarily from the perspective of reductive dehalogenation of toxic compounds, anaerobic photosynthesis and wastewater treatment, but remain relatively little studied compared to their close relatives within the larger Terrabacteria group, including Cyanobacteria, Actinobacteria, and Firmicutes. Here, we conducted a detailed phylogenetic analysis of the phylum Chloroflexota, the phylogenetically proximal candidate phylum Dormibacteraeota, and a newly defined sibling phylum proposed in the current study, Eulabeiota. These groups routinely root together in phylogenomic analyses, and constitute the Chloroflexi supergroup. Chemoautotrophy is widespread in Chloroflexi. Two Form I Rubisco ancestral subtypes that both lack the small subunit are prevalent in ca. Eulabeiota and Chloroflexota, suggesting that the predominant modern pathway for $\mathrm{CO}_{2}$ fixation evolved in these groups. The single subunit Form I Rubiscos are inferred to have evolved prior to oxygenation of the Earth's atmosphere and now predominantly occur in anaerobes. Prevalent in both Chloroflexota and ca. Eulabeiota are capacities related to aerobic oxidation of gases, especially $\mathrm{CO}$ and $\mathrm{H}_{2}$. In fact, aerobic and anaerobic $\mathrm{CO}$ dehydrogenases are widespread throughout every class-level lineage, whereas traits such as denitrification and reductive dehalogenation are heterogeneously distributed across the supergroup. Interestingly, some Chloroflexota have a novel clade of group $3 \mathrm{NiFe}$ hydrogenases that is phylogenetically distinct from previously reported groups. Overall, the analyses underline the very high level of metabolic diversity in the Chloroflexi supergroup, suggesting the ancestral metabolic platform for this group enabled highly varied adaptation to ecosystems that appeared in the aerobic world.
\end{abstract}

\section{Introduction}

The phylum Chloroflexota is represented by a variety of isolated bacteria and is one of the phyla best studied by classical approaches ${ }^{[1][2]}$. Based primarily on cultivated strains, the phylum Chloroflexi was subdivided into Anaerolineae, Ardenticatenia, Caldilineae, ca. Thermofonsia, Limnocylindria, Chloroflexia, Dehalococcoidia, Ktedonobacteria, Tepidiformia, Thermoflexia, and Thermomicrobia ${ }^{[3]}$.

The ability to reconstruct draft genomes from metagenomes circumvents the cultivation requirement and has greatly expanded the genomic coverage of the Chloroflexota and related 
bacteria. Two distinct groups represented by genomes from metagenomes are the ANG-CHLX, first reported from soil in northern California ${ }^{[4]}$ and now renamed as Dormibacteraeota ${ }^{[5]}$, and RIF-CHLX, first reported from an aquifer adjacent to the Colorado River near the town of Rifle, $\mathrm{CO}^{[6][34]}$ and later designated as Chloroflexota class ca. Limnocylindria ${ }^{[7]}$. These two groups remain relatively little explored, both from the perspective of their phylogenetic placement and metabolic traits. We use the term Chloroflexi supergroup to refer to the Chloroflexota and the RIF-CHLX. Whether Dormibacteraeota are monophyletic with Chloroflexi and RIF-CHLX and thus part of the supergroup has remained uncertain, although phylogenetic analyses suggest the Chloroflexi supergroup and Dormibacteraeota are phylogenetically proximal, and so they are included in our analyses.

Within the Chloroflexi supergroup are organisms that are obligate $\mathrm{H}_{2}$-dependent haloalkane-reducers of the class Dehalococcoidia ${ }^{[8]}$, spore-forming members with actinomycetes-like morphology of the class Ktedonobacteria ${ }^{[9] 15]}$, and the photosynthetic, thermophilic members of the class Chloroflexia ${ }^{[1]}$. Chloroflexus aurantiacus (class Chloroflexia), the type species of the phylum, was first identified from hot springs in $1974^{[1]}$; their production of bacteriochlorophyll gave them a characteristic green color, thus the name Chloroflexi, from greek ' $x \lambda$ wpós', meaning 'green'. Culturing $C$. aurantiacus in the absence of light yields an orange culture, thus the species name aurantiacus, from the latin 'aureum' for 'orange'. Newly characterized Chloroflexota have been observed which contain Type I photosystem reaction centers $^{[10]}$, although with the exception of these new organisms, all other phototrophic Chloroflexota use Type II pufLM-type photosystem reaction centers. The majority of identified microorganisms belonging to the phylum Chloroflexota, however, lack the capacity to perform photosynthesis. An interesting recent report demonstrated the genomic capacity for production of photosynthetic reaction centers in the Chloroflexota order Aggregatilineales, within the clade Anaerolinea, based on the presence of divergent pufLM-like reaction centers ${ }^{[12]}$. Some representatives of the Chloroflexi supergroup have the ability for chemoautotrophic fixation of $\mathrm{CO}_{2}{ }^{[13]}$, oxidation of $\mathrm{CO}^{[14][16]}$, and oxidation of $\mathrm{H}_{2}{ }^{[16][17]}$.

A surprising and interesting recent finding is that bacteria within Chloroflexota class Anaerolinea have Form I Rubisco sequences which form a clade that is clearly basal to previously known Form I Rubiscos. Form I Rubisco is the enzyme at the heart of the Calvin Benson Bassham (CBB) cycle used by organisms including Cyanobacteria, algae and plants in the fixation of $\mathrm{CO}_{2}$. It is considered to be one of the most abundant proteins on the planet, and one of the most important from the perspective of biosphere primary production. Notably, the new clade, referred to as Rubisco Form l', lacks the small subunit that is required for function of Form I Rubisco. Importantly, the enzyme has been biochemically characterized and its function in the CBB pathway confirmed ${ }^{[13]}$. Based on this research it was suggested that the small subunit evolved to stabilize the octamer when it adapted to an oxygenated atmosphere to increase specificity for $\mathrm{CO}_{2}$ over $\mathrm{O}_{2}{ }^{[13]}$. Form I' Rubisco has not been found in bacteria outside of the Anaerolinea, suggesting that Form I Rubisco evolved within a lineage closely related to the ancestor of the modern Chloroflexi from a single subunit form similar to l'. Better understanding of the forms and distributions of Rubisco in the Chloroflexi supergroup may provide further insight regarding the evolution of the CBB cycle and clues to the metabolic context into which it evolved. 
Here, we assembled a Chloroflexi supergroup genome dataset comprising publicly available and newly reconstructed genomes from groundwater, river sediment and soil environments. We constructed a detailed phylogeny for the supergroup, clarified the relationships among currently described groups and provided a foundation for the analysis of the distribution of metabolic traits across the lineages. Our results highlight several traits differentially distributed among phylum- and class-level lineages within the Chloroflexi supergroup and provide the most detailed phylogenetic analysis of the Chloroflexi supergroup to date.

\section{$\underline{\text { Results }}$}

\section{Ribosomal phylogeny of the Chloroflexi supergroup reveals major subdivisions}

We constructed a database that includes 2086 publicly available (see Methods) and 75 newly reconstructed genomes from the Chloroflexi supergroup. (Supplementary Table S1). A phylogenetic tree based on a concatenation of 16 ribosomal protein sequences from 2069 genomes (Fig. 1) reveals multiple potential unstudied class-level lineages within the phylum Chloroflexota (Supplementary data S1) (Table 1: Number of genomes per lineage in the Chloroflexi supergroup as assigned by phylogeny). Proximal to the Chloroflexota are two phylum-level lineages, one of which is ca. Dormibacteraeota. Some genomes from the second lineage had previously been considered to form a class within Chloroflexota, referred to in separate sources as ellin6529 (GTDB $)^{[18][19]}$, Edaphomicrobia ${ }^{[20]}, \quad$ RIF-CHLX ${ }^{[6]}$, and Limnocylindria $^{[7]}$. For this new and now clearly genomically resolved phylum-level lineage we propose the name Eulabeiota, from ancient greek Eulabeia ( $\varepsilon \cup \lambda \alpha \beta \varepsilon ı$, "timidity", "reverence" or "caution"). Genomes from ca. Dormibacteraeota have been recovered from soil and permafrost, whereas genomes from ca. Eulabeiota were obtained from diverse environments such as hydrothermal vents, freshwater, groundwater, permafrost, soil, and aquifer sediment.

Within the phylum Chloroflexota are four well sampled, deeply branching clades, some of which contain multiple classes but form cohesive phylogenetic groups: the Chloroflexia, Anaerolinea, Ktedonobacteria, and Dehalococcoidia. We present new genomes for all four clades. Additionally, rooting proximal to the Ktedonobacteria are two deeply branching, poorly sampled lineages, one of which is composed entirely of genomes obtained from coral and sponge holobiont metagenomes ${ }^{[21][34]}$.

Within the class Dehalococcoidia are three major groups. Interestingly, two of these groups lack essentially all of the genes necessary for the synthesis of peptidoglycan (Fig. 2). This was previously noted for a few cultivated representatives ${ }^{[22]}$ but has not previously been evaluated through an analysis of all publicly available genomes of this clade. One clade within Dehalococcoidia includes Dehaloccoides mccartyi, which has an S-layer like protein cell wall instead of a wall containing peptidoglycan ${ }^{[23]}$. Another group within Dehalococcoidia is the SAR202 group, primarily comprised of representatives from marine environments ${ }^{[24][25]}$.

Some genomes basal to the Dehalococcoidia clade, as well as genomes within the more distal Dehalococcoidales clade, contain genes which code for the Wood-Ljungdahl pathway as 
previously reported ${ }^{[26]}$. The SAR202 clade and the rest of the Chloroflexi supergroup lack the genes necessary for the full Wood-Ljungdahl pathway.

\section{Rubisco}

Ribulose 1,5-bisphosphate carboxylase/oxygenase is widespread in phototrophic and non-phototrophic members of the Chloroflexi supergroup. We identified no cases of form II or form II/III Rubisco in the supergroup. All Rubisco large chain $(r b c L)$ genes from members of the Chloroflexi supergroup are phylogenetically related to form I Rubisco, and form I rbcL sequences are found in genomes belonging to all clades analyzed in this study, including ca. Dormibacteraeota and ca. Eulabeiota. However, one sequence from a groundwater-associated Eulabeiota genome and one from a sediment-associated Eulabeiota genome occur without $r b c S$, the small subunit canonically found alongside the large chain. These two sequences indicate a clade that is basal to recently reported divergent Anaerolinea form 1' Rubiscos that also lack a small subunit ${ }^{[13]}$. No rbcS sequences were found anywhere in the Eulabeiota genomes containing these novel rbcL sequences. The Eulabeiota $r b c L$ sequences display low-level homology to both form III and form I rubisco sequences, at approximately $50 \%$ identity to form III representatives and $48 \%$ identity to form I representatives by blastP, and cluster with form I representatives in the phylogeny. However, unlike form III rubisco, the Anaerolinea form 1' Rubiscos and both Eulabeiota rbcL genes are encoded adjacent to phosphoribulokinase ( $p r k B$ ) and $c b b R$, a transcriptional regulator of the rubisco operon (Fig. $\mathbf{3}$ ).

To further investigate the diversity and environmental distribution of Rubisco with sequences related to those from Eulabeiota, we searched a large dataset of unbinned metagenomic data and found examples in datasets from soils, a salt pond and groundwater. (Fig. 3). Phylogenetic analysis using a dataset that included these unbinned $r b c L$ sequences, the two Eulabeiota sequences and sequences form 1' Rubiscos establish that they form a clade basal to both form 1' and form I and clearly separate from form III Rubisco. (Fig. 3a). We refer to this new clade of Rubisco as form I- $\alpha$. These genomes encode for a partial CBB pathway, and both PRK and PGK are present in these genomes. Given clear phylogenetic affiliation with form I Rubisco, the co-occurrence of the binned and some unbinned sequences with prkB and $c b b R$, recent biochemical evidence that form I' fix $\mathrm{CO}_{2}$ despite the absence of $r b c S$, and the presence of PRK and PGK enzymes in the genomes where form 1- $\alpha$ is detected, we conclude that the Eulabeiota form $1-\alpha$ clade are involved in $\mathrm{CO}_{2}$ fixation via the CBB pathway. Additionally, analysis of unbinned $r b c L$ sequences revealed a putative clade proximal to form I' and form I, which we have designated form I".

\section{Photosynthesis}

The majority of known phototrophic organisms within the phylum Chloroflexota are within the class Chloroflexia, and use type II photosystem reaction centers along with bacteriochlorophyll to perform non-oxygenic photosynthesis ${ }^{[11]}$. Chloroflexota genomes containing type I photosystem reaction centers have been recently reported ${ }^{[10]}$, and we observed 
these genes in the genomes deposited to NCBI from this study, but no additional type I photosystem reaction center hits were observed in other genomes from the Chloroflexi supergroup. Here we present 6 newly reported genomes from the Anaerolinea clade with type II photosystem reaction centers (pufLM), and identify 7 Anaerolinea genomes with pufLM from public data. These photosystem reaction centers show phylogenetic proximity to sequences from the clade Chloroflexia, yet separate into clades distinct from sequences of class Anaerolinea. Chloroflexia and Anaerolinea genomes contain pufLM fusion genes, as reported previously ${ }^{[11]}$ (Fig. 4). Alongside the type II photosystem reaction centers, these genomes contain homologs to photosystem reaction center-associated cytochromes and transcription factors. Additionally, they contain light harvesting proteins associated with the bacteriochlorophyll binding proteins of the chlorosome found in photoautotrophic Chloroflexia ${ }^{[27]}$, suggesting that these organisms are capable of performing photosynthesis. These genomes also have at least partial bacteriochlorophyll biosynthesis pathways.

\section{Carbon compound utilization}

Members of the Chloroflexi supergroup vary in their predicted capacities to process carbohydrate compounds. For example, Anaerolinea genomes have, on average, nearly 14 times more predicted glycosyltransferases per genome than those of Dehalococcoidia (Fig. 5). Anaerolinea and Chloroflexia are enriched in carbohydrate metabolism genes of all CAZy (Carbohydrate-Active enZyme) classes compared to other phyletic groups. Numerous Dehalococcoidia, including members of the SAR202 group, and ca. Dormibacteraeota have genomes that encode for very few carbohydrate metabolizing enzymes.

\section{Hydrogenases}

We investigated the distribution of capacities related to hydrogen metabolism across the Chloroflexi supergroup. To enable predictions, hydrogenases were classified into types using phylogenetic analyses based on the sequences of the large subunit. Detailed information on the hydrogenases observed in genomes from the Chloroflexi supergroup is available in Supplementary table S3 and in Supplementary Text S4. Hydrogenases belonging to FeFe group C, FeFe group B, and energy-converting hydrogenase-related (Ehr) complexes are reported here for the first time in the Chloroflexi supergroup.

Overall, we find that hydrogenases are abundant, especially in Anaerolinea and Dehalococcoidia, which are typically found in environments that are anaerobic or periodically anaerobic. The function of these hydrogenases can be $\mathrm{H}_{2}$ consumption, utilization, or both. In 
contrast, the Eulabeiota and Ktedonobacteria have relatively few hydrogenase hits per genome. The hydrogenases observed within the Ktedonobacteria generally belong to NiFe group $1 \mathrm{~h}$, which have been observed to oxidize $\mathrm{H}_{2}$ at atmospheric concentrations in the presence of $\mathrm{O}_{2}{ }^{[30]}$.

A recently described group of NiFe hydrogenases, group 11, are found exclusively in the Eulabeiota. These have been hypothesized to provide electrons to Rubisco and support carbon fixation [13]. Consistent with this, the majority of genomes containing group 1 I NiFe hydrogenases also contain form I Rubisco (with the small and large subunits), although the hydrogenase and rbc gene clusters are not co-localized in these genomes.

The most numerically abundant hydrogenase subtypes within the Chloroflexi supergroup are $\mathrm{NiFe}$ group 3, including a novel NiFe group 3c hydrogenase implicated in electron bifurcation, and group $3 \mathrm{~d}$ NiFe hydrogenases, which usually couple reversible $\mathrm{H}_{2}$ oxidation to $\mathrm{NAD}^{+}$reduction (Fig. 6). In several Anaerolinea and Dehalococcoidia genomes, NiFe group 3c hydrogenase was preceded by genes encoding a heterodisulfide reductase (HdrABC), and in some cases electron transfer flavoprotein (ETF) complex. A complex between HdrABC and hydrogenase has been implicated in flavin-based electron bifurcation in Archaea, though it requires a third protein partner ${ }^{[28][29]}$. An association between group 3c NiFe hydrogenase, EtfAB, and an heterodisulfide reductase (HdrA2B2C2D) has the potential to perform electron bifurcation (see Supplementary Text $\mathbf{S 4}$ for details).

Unique to the Anaerolinea is a divergent clade of NiFe group 3 hydrogenases for which the large subunit is phylogenetically proximal to groups $3 \mathrm{c}$ and $3 \mathrm{~d}$. For this group, we propose the group name $3 e$. This hydrogenase is accompanied by a small subunit but lacks both identified accessory proteins and electron transfer subunits, and may interact with unknown partners.

In all groups, we identified hydrogenases that likely support $\mathrm{H}_{2}$-oxidation-based energy generation. Other hydrogenases, especially those associated with the cell membrane (NiFe type 4), are likely involved in proton translocation and were found across the Chloroflexi supergroup, with the exception of the Dormibacteraeota. (Supplementary figure S3, Supplementary Text S4)

\section{Multiheme Cytochromes}

Multiheme cytochrome proteins, defined as having $\geq 4$ heme-binding motifs, are especially abundant in Anaerolinea. Of all proteins with $\geq 20 \mathrm{CxxCH}$ heme-binding motifs in the Chloroflexi supergroup, the majority (88 of 124) occur in genomes from the Anaerolinea. Large multiheme cytochromes with $\geq 20$ heme-binding motifs are understood to participate in redox reactions, for example iron and manganese reduction reactions ${ }^{[31]}$, and are hypothesized to play a role in extracellular respiration in iron reducing bacteria ${ }^{[32]}$. Many of the large multiheme cytochromes contain transmembrane domains and demonstrate homology to models trained on multiheme cytochrome proteins from the genus Geobacter ( $\mathrm{GSu} \_\mathrm{C} 4 \times \mathrm{C} \_\mathrm{C} 2 \times \mathrm{CH}$ ), noted for its ability to participate in extracellular metal ion transformations. This suggests that Anaerolinea use multiheme cytochromes to deliver electrons to an extracellular terminal electron acceptor. 


\section{Nitrogen cycling}

Within the Chloroflexi supergroup, genes encoding for nitrogen transformation pathways are common but unevenly distributed phylogenetically (Fig. 7). Several nitrogen transformation capacities were not detected in the Chloroflexi supergroup, including ammonia oxidation (amo $A B)$, nitrite oxidation by nas $A B$, and $\mathrm{N}_{2} \mathrm{O}$ reduction by norBC.

Reduction of nitrate and nitrite are the most commonly observed nitrogen transformation capacities in the Chloroflexi supergroup; all studied clades contained representatives capable of reducing nitrite. Only ca. Dormibacteraeota lack the genomic capacity to perform assimilatory or dissimilatory nitrate reduction. There are many Anaerolinea and Dehalococcoidia that can reduce nitrogen-containing compounds, and via many mechanisms. In contrast, Dormibacteraeota encode relatively few genes for nitrogen compound transformations, and a low diversity of pathways.

Some Anaerolinea genomes contain multiple genes for reduction of $\mathrm{NO}_{3}{ }^{-}$to $\mathrm{NO}_{2}^{-}$. They contain the narGHIJ nitrate reductase complex, periplasmic nitrate reductase (napAB), which is part of the dissimilatory pathway, and ferredoxin nitrate reductase (narB), which is involved in assimilatory nitrate reduction. The narGHIJ system is also present in genomes from the candidate phyla Dormibacteraeota and Eulabeiota, but was not detected in class Chloroflexia or Ktedonobacteria.

Reduction of $\mathrm{NO}_{2}^{-}$to $\mathrm{NH}_{3}$ through the $n r f A H$ denitrification system is very common within the Anaerolinea. This pathway was identified even where genes for nitrate reduction were not identified elsewhere in the genome, suggesting that these organisms rely on nitrite produced by coexisting community members.

Nitrite reduction via nirA, associated with the assimilatory pathway, is present in all clades of the phylum Chloroflexota but absent from the candidate phyla Dormibacteraeota and Eulabeiota. The capacity for $\mathrm{NO}_{2}^{-}$reduction to $\mathrm{NO}$ via nirk or nirS is common across the supergroup. The capacity to reduce $\mathrm{NO}$ to $\mathrm{N}_{2} \mathrm{O}$ via norBC is only observed in the Dehalococcoidia. Further reduction of $\mathrm{N}_{2} \mathrm{O}$ to $\mathrm{N}_{2}$ via nos $\mathrm{Z}$ is common across the Chloroflexi supergroup, but absent from the Ktedonobacteria and ca. Dormibacteraeota.

Nitrogen fixation is present in the classes Dehalococcoidia and Chloroflexia. Notably, there are multiple phylogenetically distinct forms of nifH (Supplementary Fig. S4). The Dehalococcoidia containing nifH are distal to those found in Chloroflexia and cluster with group II nifH sequences, whereas nitrogenases from class Chloroflexia form a clade separate from previously defined subtypes basal to form I nifH. All of the nifH sequences observed in genomes also containing nifA and nifB are further implicated in nitrogen fixation by phylogenetic proximity to biochemically verified sequences. 


\section{The Sulfur Cycle}

Microorganisms from the Chloroflexi supergroup are well known to participate in biogeochemical sulfur cycling ${ }^{[32]}$, but the distribution of sulfur cycle genes throughout the supergroup has not been reported. Our analysis indicates that the most common genes are implicated in assimilatory sulfate reduction. The sat sulfate adenylyltransferase system, which can be involved in both assimilatory and dissimilatory steps, is common throughout the Chloroflexi supergroup. An alternative gene for this reaction, sulfate reductase, PAPSS/K13811, is found primarily in genomes of ca. Dormibacteraeota, possibly as part of the assimilatory sulfate reduction pathway. The assimilatory reduction of $\mathrm{SO}_{3}{ }^{2-}$ to $\mathrm{H}_{2} \mathrm{~S}$ via the cyslJ and sir sulfite reductase systems seems to be particularly common among the Eulabeiota and Ktedonobacteria, but absent from Dormibacteraeota.

The reduction of adenylylsulfate to $\mathrm{SO}_{2}^{-}$via aprAB in the dissimilatory sulfate reduction pathway is rare and sparsely distributed in the Chloroflexi supergroup. The $d s r A B$ genes, which can both reduce sulfite and oxidize sulfide, are rare. Sulfur oxygenase-reductase were identified in two Anaerolinea genomes, but this gene is absent from the rest of the supergroup. Thiosulfate oxidation via the sox complex was not predicted for any bacteria of the Chloroflexi supergroup.

Dimethyl sulfoxide (DMSO) reductase genes of the $d m s A B C$ family were detected in all lineages of the Chloroflexi supergroup with the exception of the Dormibacteraeota, although genes that would indicate metabolism of the DMSO breakdown product dimethyl sulfide (DMS), such as dimethyl-sulfide monooxygenase $(d m o A)$ and dimethyl sulfide dehydrogenase $(d d h A B C)$, were not detected in this dataset. Undetected in the dataset were genes implicated in the breakdown of dimethylpropiothetin (DMSP, a compatible solute) to form DMS. The only gene present in the pathway that converts DMS ultimately to sulfate (e.g. sox and sor) is methanethiol oxidase, which produces sulfide from methylmercaptan, and is particularly abundant in the Dormibacteraeota and Ktedonobacteria.

\section{CRISPR-Cas Systems}

The abundance of CRISPR-Cas phage defense systems within the Chloroflexi supergroup is highly dependent upon the environment of origin and organism taxonomy (Supplementary Figure S5). The candidate phyla Dormibacteraeota and Eulabeiota have a strikingly low average number of detected CRISPR arrays relative to other groups, although intact cas protein cassettes are detected in genomes from these candidate phyla which otherwise lack detectable CRISPR arrays. 


\section{Discussion}

Taxonomic databases disagree on the number of subdivisions within the Chloroflexi (alternatively called the Chloroflexota). For simplicity, we used the long-established phylogenetically cohesive class-level subdivisions Anaerolinea, Dehalococcoidia, Ktedonobacteria, and Chloroflexia as the foundation for our further taxonomic analyses. Our ribosomal phylogeny clearly supports the existence of these four main groups. A poorly sampled lineage which lies proximal to the Ktedonobacteria (Fig. 1) is comprised entirely of endosymbionts of sponges and corals ${ }^{[21][34]}$.

External to the Chloroflexota lie the candidate phylum Dormibacteraeota (previously Candidate Division AD3) and another distinct lineage that includes groups previously named Limnocylindria and Edaphomicrobia, additionally demarcated by GTDB as class Ellin6529 within the phylum Chloroflexota. The Limnocylindria and Edaphomicrobia were recently proposed classes within the Chloroflexota. Our analyses indicate that the Limnocylindria and Edaphomicrobia are closely related, part of a single phylum-level lineage, and that they place outside of the Chloroflexota. Our newly reported genomes clarify their phylogeny and substantially expand the breadth of the lineage that includes the newly proposed candidate phylum ca. Eulabeiota. Together, the Chloroflexota, Eulabeiota and Dormibacteraeota comprise the Chloroflexi supergroup, which is part of the larger Terrabacteria group.

\section{Eulabeiota}

The Eulabeiota were first named Ellin6529 after an isolate was obtained from agricultural soil near Ellinbank, Victoria, Australia ${ }^{[36]}$. Unfortunately, the isolate was lost. However, a full-length 16S rRNA sequence was obtained and used to study the abundance of this lineage across habitats. Although the majority of Eulabeiota sequences we studied came from soils, often from cold or perennially dry environments, they also appear in freshwater environments, such as partially melted permafrost ${ }^{[5]}$, lakes ${ }^{[7]}$, and groundwater ${ }^{[38][39][6]}$ [Christine's paper, BJP, relevant Rifle publications]. Notably, genomes from the Eulabeiota have been obtained from marine oil seeps ${ }^{[81]}$ and one from a hydrothermal vent ${ }^{[37]}$.

The Eulabeiota lack outer membrane synthesis genes and contain similar peptidoglycan biosynthesis machinery to their sister phyla Chloroflexota and Dormibacteraeota as well as more distant neighbors such as the Actinobacteria and Armatimonadetes, suggesting that the Eulabeiota are monoderm organisms.

Nearly all Eulabeiota genomes encode at least one type of cytochrome $c$ terminal oxidase, indicating that they are at least facultatively aerobic. However, some genomes lack terminal oxidases, and have markers for anaerobic metabolism such as anaerobic $\mathrm{CO}$ dehydrogenase (coos), suggesting that some Eulabeiota have adapted to strictly anaerobic environments.

Some Eulabeiota genomes encode the capacity for $\mathrm{CO}_{2}$ fixation via the CBB pathway using Rubisco ( $r b c L)$. Interestingly, phylogenetic analysis separates the Rubisco genes present in the Eulabeiota into multiple clades, one of which, designated form I- $\alpha$, lacks the small subunit $r b c S$ (Fig. 3). Both genomes with the $\mathrm{I}-\alpha$ rbcL were obtained from aquifer water and sediment 
from Rifle, Colorado ${ }^{[6]}$, in an environment with low dissolved oxygen content. This Rubisco clade is more deeply branching than the other recently reported single subunit form l' Rubisco, which was shown biochemically to function as an oxygen-sensitive carboxylase enzyme in the absence of $r b c S^{[13]}$. We infer that $\mathrm{I}-\alpha$ rbcL is similar in structure and function to the precursor of modern Rubisco found in cyanobacteria, algae, and plants. Consistent with the suggestion for form I' Rubisco, we infer that the Eulabeiota form I- $\alpha$ evolved to function in an anaerobic world and that this precursor gave rise to the two subunit form I complex that was widely laterally transferred across the tree of life after the rise of $\mathrm{O}_{2}$ in the atmosphere.

\section{Dormibacteraeota}

The recently characterized candidate phylum Dormibacteraeota has thus far been found exclusively in soil and permafrost metagenomes, and has no cultured representatives. This clade seems to be comprised of at least facultatively aerobic monoderm organisms, as evidenced by a widespread distribution of aerobic $\mathrm{CO}$ dehydrogenase gene cassettes as well as cytochrome $\mathrm{C}$ terminal oxidase genes throughout the phylum. Hydrogen metabolism is also abundant, with Dormibacteraeota genomes containing type 1 and type 3 NiFe hydrogenases, although type 1 and type 3 NiFe hydrogenase subtypes are not observed together in a single genome from this clade (Supplementary Table S3).

\section{Dehalococcoidia}

The Dehalococcoidia contains three major phylogenetic subdivisions that exhibit different sets of metabolic pathways and have characteristic environmental distribution patterns (Fig. 2). We will refer to these three subdivisions as the basal clade, the SAR202, and the Dehalococcoides clade (Fig. 2).

Notable is the absence of a functional peptidoglycan biosynthesis pathway in a substantial portion of theSAR202 and Dehalococcoides clades. However, genomes in the basal clade often have peptidoglycan biosynthesis pathways. Previous studies ${ }^{[8]}$ have highlighted a lack of peptidoglycan in Dehalococcoides mccartyi, the type species of the Dehalococcoidia, although microscopy reveals a cell wall-like structure perhaps similar in function to those with S-layers found in other bacteria and archaea.

Some genomes within the Dehalococcoides lack F-type ATPases, instead containing only a V-type ATPase, as has been previously reported ${ }^{[26]}$. The genomes that contain $\mathrm{V}$-type and lack F-type ATPases, along with a small subset of other Dehalococcoides genomes, lack genes for the NADH-quinone oxidoreductase complex, indicating the use of substrate-level phosphorylation to generate ATP.

The functional division within the class Dehalococcoidia also correlates with the environments of origin. The SAR202 clade are primarily from marine or saline environments, whereas genomes from the Dehalococcoides and basal clades are generally from terrestrial sources such as groundwater, soil, and freshwater, although there are exceptions in each case.

\section{Anaerolinea}


The most well-represented clade within the dataset is the Anaerolinea. They have extensive capacity for nitrogen metabolism, some are likely photosynthetic and some are capable of assimilatory and dissimilatory sulfate reduction. Many are predicted to be autotrophs, using both Form I and Form 1' Rubisco in the CBB pathway, and Form 1' is exclusively found in genomes which fall in this clade. Many Anaerolinea genomes encode numerous multiheme cytochromes, some of which have $>20$ heme-binding motifs per protein. These may be involved in extracellular electron transfer reactions, including metal reduction and oxidation ${ }^{[88][89]}$.

Photosystem II reaction centers phylogenetically distinct from those found in the Chloroflexia are observed in genomes from the Anaerolinea. These genomes contain partial bacteriochlorophyll synthesis pathways but include crucial genes such as bchP, which catalyzes the last reaction in the biosynthesis of bacteriochlorophyll $c$, suggesting that although the full bacteriochlorophyll synthesis pathway is not detected in these genomes that the synthesis of bacteriochlorophyll does occur, either using divergent bacteriochlorophyll synthesis genes or by obtaining precursor compounds from other bacteria in their communities. These putatively photosynthetic Anaerolinea are primarily found in hot spring environments, although three such genomes were obtained from stromatolite metagenomes ${ }^{[12][40]}$.

The Anaerolinea contain a number of unique subtypes of NiFe hydrogenases, including the phylogenetically divergent group $3 \mathrm{e}$, which is unique to the Anaerolinea, and a variant of group $1 \mathrm{f}$ which lacks a cytochrome subunit but associates with a nrfD-like molybdopterin subunit. They are the only clade within the Chloroflexi supergroup to contain FeFe hydrogenases of type B. Anaerolinea genomes also contain hydrogenases which are proximal to heterodisulfide reductase complexes as well as electron transfer flavoprotein subunits, potentially implicating these organisms in electron bifurcation. The diversity in the number of observed hydrogenase subtypes in the Anaerolinea, as well as the unique hydrogenase subtype of group $3 e$ observed exclusively therein, points to the importance of hydrogen-based metabolism for members of this lineage, and suggests that members of this clade are well-adapted to at least periodically anaerobic environments.

Anaerolinea have the most varied environmental distribution of any clade in this study, with collection temperatures ranging from hot springs and hydrothermal vents ${ }^{[41]}$ to permafrost ${ }^{[5]}$ and the human oral microbiome (BioProject PRJNA282954). They are common in soils and occur in activated sludge from wastewater treatment plants ${ }^{[42]}$, where they contribute to sludge flocculation. Their notably extensive metabolic diversity may in part reflect their adaptation to very diverse environments, and the associated varied energy resources.

\section{Chloroflexia}

The Chloroflexia group is well-studied, and contains the class Chloroflexia, the type class of the Chloroflexota. The group contains the majority of the phototrophic organisms within the phylum, and is unique among the clades of the Chloroflexi supergroup in that several are capable of performing $\mathrm{CO}_{2}$ fixation via the 3-hydroxypropionate bicycle, as has been previously observed $^{[43]}$. Genomes from this group, especially those most closely related to Chloroflexus aggregans, the photoautotrophic type species of the phylum, tend to be observed most often in 
hot springs or freshwater sources. More divergent lineages within the group basal to the Chloroflexia are more often found in soil, and lack genes coding for anoxygenic photosynthetic reaction centers as well as the complete 3-hydroxypropionate bicycle, suggesting they occupy different metabolic niches despite their phylogenetic proximity to typical Chloroflexia.

\section{Ktedonobacteria}

The Ktedonobacteria group is comprised of two phylogenetically distinct subdivisions, one of which is comprised entirely of genomes obtained from soil samples and includes the type genus Ktedonobacter, and another comprised of genomes primarily from stratified freshwater lakes and ponds ${ }^{[4]}$ and groundwater sources ${ }^{[38]}$. Ktedonobacteria genomes are of particular research interest because of their size and the large number of secondary metabolite gene clusters. This extends prior findings ${ }^{[45][46]}$ which show that substantial inventories of secondary metabolite gene clusters in genomes obtained from soil. Products of this type of metabolism may be implicated in interaction among organisms or between organisms and their environment.

Ktedonobacteria contain fewer hydrogenase subtypes than other clades, and the majority of the observed subtypes in this clade belong to the $\mathrm{O}_{2}$-tolerant type $1 \mathrm{~h}^{[30]}$. Hydrogen oxidation is likely important in anaerobic (e.g., groundwater) or seasonally anaerobic soils where fermentation generates $\mathrm{H}_{2}$. Members of this group also contain form I rbcL sequences which coincide with rbcS and phosphoribulokinase genes in the same genome, strongly suggesting that these organisms are capable of carboxydotrophy.

\section{Conclusion}

The Chloroflexi supergroup is comprised of bacteria from across diverse environments, and is largely understood by way of genome-resolved metagenomics. Our study provides the most detailed ribosomal phylogeny of the Chloroflexi supergroup to date, which allowed for an investigation of the distribution of important functional genes in clades throughout the supergroup and revealed functional differences between and within class- and phylum-level lineages within the supergroup. Many genomes belonging to the Chloroflexi supergroup contain novel genomic features, which are specific to particular clades within the supergroup and contain phylogenetically novel representatives of well-studied protein families such as Rubisco and NiFe hydrogenases. Our work highlights the diversity and ubiquity of hydrogen-dependent metabolism in the Chloroflexi supergroup and reveals phylogenetically novel clades of putative hydrogenases of type $3 \mathrm{e}$ which have thus far only been observed in genomes belonging to the supergroup. Additionally, we report for the first time the phylogenetic distribution of multiple Chloroflexi supergroup-exclusive clades of form I-like Rubisco as first reported in Banda et al. ${ }^{[13]}$, including a new form of putative Rubisco designated form I- $\alpha$. Biochemical investigation of novel proteins such as form I- $\alpha$ Rubisco and group 3 e NiFe hydrogenase is crucial to understand the potentially significantly altered catalytic function of these groups relative to biochemically 
bioRxiv preprint doi: https://doi.org/10.1101/2021.08.23.457424; this version posted August 24,2021 . The copyright holder for this preprint

(which was not certified by peer review) is the author/funder, who has granted bioRxiv a license to display the preprint in perpetuity. It is made available under aCC-BY-ND 4.0 International license.

characterized clades. Our results should guide targeted cultivation efforts and aid further investigations into these ubiquitous and understudied organisms. 


\section{Methods}

\section{Database Construction}

Genomes were downloaded from three databases: NCBI GenBank ${ }^{[47]}$, PATRIC ${ }^{[48]}$, and ggKbase (https://ggkbase.berkeley.edu/). All database downloads were performed on August 15, 2020. Those downloaded from NCBI GenBank were downloaded using a custom script (attached, supplemental) which utilizes the NCBI Entrez python API, and searched for all genbank genomes with hits to 'Chloroflexi'. Genomes from BioProjects less than two years old and without associated publications were discarded. Genomes from PATRIC were gathered by searching the keywords 'Chloroflexi' and 'AD3', the former name for the Dormibacteraeota, on PATRIC and downloading all resulting genomes on Dec. 13, 2019. Genomes from ggKbase were downloaded using only genomes with taxonomic hits to Dormibacteraeota (ANG-CHLX), Eulabeiota (RIF-CHLX), or Chloroflexota. Information on the originating database and additional metadata for each genome can be found in Supplementary Table S1.

Genomes from all sources were then dereplicated using $\mathrm{dRep}^{[49]}$ using a $100 \%$ identity dereplication threshold, additionally removing genomes with greater than $25 \%$ checkM $^{[50]}$ contamination and less than $75 \%$ checkM completeness.

Genomes were annotated using KOFAMScan ${ }^{[51]}$, applying provided bitscore thresholds. KOFAMscan hits were then counted using a custom python script. Counts were normalized using the Hellinger transformation ${ }^{[52]}$ prior to projection with Uniform Manifold Approximation and Projection (UMAP) ${ }^{[53]}$. Genomes were additionally annotated using USEARCH ${ }^{[84]}$ against Uniprot [85], Uniref90 and KEGG ${ }^{[86]}$, and $16 \mathrm{~S}$ rRNA and tRNAs predicted as described in Diamond et al. [4].

All phylogenetic trees included in this paper were visualized using the Interactive Tree of Life (iTOL) $)^{[54]}$.

Newly presented genomes in this study were obtained from four projects and processed using the ggKbase annotation and binning pipeline. Sampled locations include: hot springs in Tibet and Yunnan province, China; deep boreholes in Japan (BJP); soil samples taken from the East River watershed in Colorado, United States; and a series of anammox and dechlorination bioreactors.

\section{Analyses for samples obtained from the Borehole Japan Project}

Genomes from the Borehole Japan project were obtained from $\sim 439 \mathrm{~L}$ of groundwater samples collected at the Horonobe Underground Research Laboratory and the Mizunami Underground Research Laboratory in Japan, according to methods outlined in Hernsdorf et al. $2017^{[39]}$ and Matheus Carnevali et al.,2019 ${ }^{[67]}$. In brief, genomic DNA was extracted from the biomass gathered on the $0.22 \mu \mathrm{m}$ GVWP filters using an Extrap Soil DNA Kit Plus version 2 (Nippon Steel and Sumikin EcoTech Corporation, Tsukuba, Japan). Genomic DNA libraries were generated using TruSeq Nano DNA sample Prep Kit (Illumina, San Diego, CA, USA) according to the manufacturer's instructions, and $150 \mathrm{bp}$ paired-end reads with a $550 \mathrm{bp}$ insert size were 
sequenced by Hokkaido System Science Co. using Illumina HiSeq 2500. Assembly and binning were performed as reported previously ${ }^{[39][67]}$.

\section{Sampling, DNA extraction, metagenomic sequencing and analyses for soil samples obtained from the East River, Crested Butte, CO.}

Genomes were obtained from soil cores sampled from the East River in Crested Butte, $\mathrm{CO}$ in 2016 and 2017. Samples were taken from two depth regimes: shallow (between $3-10 \mathrm{~cm}$ from the soil surface) and deep (between $9-20 \mathrm{~cm}$ from the soil surface). DNA was extracted from the samples using the Qiagen Powermax Soil DNA extraction kit and submitted to the Joint Genome Institute for sequencing. Soil samples were collected using sterile tools, including a soil core sampler and $7.6 \times 15.2 \mathrm{~cm}$ plastic corer liners (AMS, Inc), stainless-steel spatulas, and Whirl-pak bags. Samples were immediately stored in coolers for transportation to RMBL, where samples were prepared for archival and transportation to the University of California, Berkeley. Soil cores were broken apart and manually homogenized inside the Whirl-pak bags.

Subsamples for chemical analyses, DNA extractions, and long-term archival were obtained inside a biosafety cabinet, kept at $-80^{\circ} \mathrm{C}$, transported in dry ice, and stored at $-80^{\circ} \mathrm{C}$ at the University of California, Berkeley.

Genomic DNA was extracted from $\sim 10 \mathrm{~g}$ of thawed soil using Powermax Soil DNA extraction kit (Qiagen) with some minor modifications as follows. Initial cell lysis by vortexing vigorously was substituted by placing the tubes in a water bath at $65^{\circ} \mathrm{C}$ for $30 \mathrm{~min}$ and mixing by inversion every $10 \mathrm{~min}$ to decrease shearing of the genomic DNA. After adding the high concentration salt solution that allows binding of DNA to the silica membrane column used for removal of chemical contaminants, vacuum was used instead of multiple centrifugation steps. Finally, DNA was eluted from the membrane using $10 \mathrm{~mL}$ of the elution buffer $(10 \mathrm{mM}$ Tris buffer) instead of $5 \mathrm{~mL}$ to ensure full release of the DNA. DNA was precipitated out of solution using $10 \mathrm{~mL}$ of a 3-M sodium acetate $(\mathrm{pH} \mathrm{5.2)}$ and glycogen $(20 \mathrm{mg} / \mathrm{mL})$ solution and $20 \mathrm{~mL}$ $100 \%$ sterile-filtered ethanol. The mix was incubated overnight at $4{ }^{\circ} \mathrm{C}$, centrifuged at $15,000 \times g$ for $30 \mathrm{~min}$ at room temperature, and the resulting pellet was washed with chilled $10 \mathrm{~mL}$ sterile-filtered $70 \%$ ethanol, centrifuged at $15,000 \times g$ for $30 \mathrm{~min}$, allowed to air dry in a biosafety cabinet for 15-20 min, and resuspended in $100 \mu \mathrm{L}$ of the original elution buffer. Genomic DNA yields were between 0.1 and $1.0 \mu \mathrm{g} / \mu \mathrm{L}$ except for two samples with $0.06 \mu \mathrm{g} / \mu \mathrm{L}$. Power Clean Pro DNA clean up kit (Qiagen) was used to purify $10 \mu \mathrm{g}$ of DNA following manufacturer's instructions except for any vortexing which was substituted by flickering of the tubes to preserve the integrity of the high-molecular-weight DNA. DNA was resuspended in the elution buffer (10 $\mathrm{mM}$ Tris buffer, $\mathrm{pH} 8$ ) at a final concentration of $10 \mathrm{ng} / \mu \mathrm{L}$ and a total of $0.5 \mu \mathrm{g}$ of genomic DNA. DNA was quantified using a Qubit double-stranded broad range DNA Assay or the high-sensitivity assay (ThermoFisher Scientific) if necessary. Additionally, the integrity of the genomic DNA was confirmed on agarose gels and the cleanness of the extracts tested by absence of inhibition during PCR.

Clean DNA extracts and co-extracts were submitted for sequencing at the Joint Genome Institute (Walnut Creek, CA), where samples were subjected to a quality control check. Sequencing libraries were prepared in microcentrifuge tubes. One hundred nanograms of 
genomic DNA was sheared to 600 bp pieces using the Covaris LE220 and size selected with SPRI using AMPureXP beads (Beckman Coulter). The fragments were treated with end repair, A-tailing, and ligation of Illumina-compatible adapters (IDT, Inc) using the KAPA Illumina Library prep kit (KAPA biosystems). Libraries for the rest of the samples were prepared in 96-well plates. Plate-based DNA library preparation for Illumina sequencing was performed on the PerkinEImer Sciclone NGS robotic liquid handling system using Kapa Biosystems library preparation kit. Two hundred nanograms of sample DNA was sheared to $600 \mathrm{bp}$ using a Covaris LE220 focused-ultrasonicator. The sheared DNA fragments were size selected by double-SPRI and then the selected fragments were end-repaired, A-tailed, and ligated with Illumina-compatible sequencing adaptors from IDT containing a unique molecular index barcode for each sample library.

All the libraries were quantified using KAPA Biosystem's next-generation sequencing library qPCR kit and a Roche LightCycler 480 real-time PCR instrument. The quantified libraries were then multiplexed with other libraries, and the pool of libraries was prepared for sequencing on Illumina HiSeq sequencing platform utilizing a TruSeq paired-end cluster kit, v4, and Illumina's cBot instrument to generate a clustered flow cell for sequencing. Sequencing of the flow cell was performed on the Illumina HiSeq 2500 sequencer using HiSeq TruSeq SBS sequencing kits, v4, following a $2 \times 150$ indexed run recipe.

Methods used for metagenome assembly and annotation are described elsewhere [82]. In brief, after quality filtering, reads from individual samples were assembled separately using IDBA-UD v1.1.1 [80] with a minimum k-mer size of 40, a maximum k-mer size of 140, and step size of 20 . Only contigs $>1 \mathrm{~Kb}$ were kept for further analyses. Reads were mapped to the assemblies using Bowtie ${ }^{[55]}$ and default settings to estimate coverage.

Annotated metagenomes from both years were uploaded onto ggKbase (https://ggkbase.berkeley.edu), where binning tools based on GC content, coverage, and winning taxonomy [38] were used for genome binning. These bins and additional bins that were obtained with the automated binners ABAWACA1 (https://github.com/CK7/abawaca), ABAWACA2, MetaBAT ${ }^{[56]}$, Maxbin2 ${ }^{[91]}$, and Concoct ${ }^{[92]}$ were pooled, and DAStool ${ }^{[93]}$ was used for selection of the best set of bins from each sample as described by Diamond et al. ${ }^{[4]}$, with a completeness threshold applied of $>=70 \%$ as measured by checkM ${ }^{[50]}$.

\section{Sampling, DNA extraction, metagenomic sequencing and analyses for dechlorinating and anammox bioreactors.}

Genomes were obtained from reactors described in Lee et al. $2019^{[77]}$ and Mao et al. $2020^{[78]}$. Genomic DNA was extracted from the samples using either the Qiagen (Valencia, CA, USA) DNeasy Blood \& Tissue kit or the AllPrep DNA/RNA Mini Kit (Qiagen) according to the manufacturer's recommendations as outlined in Lee et al. 2020. Metagenomic reads were assembled with IDBA_UD ${ }^{[80]}$ with default parameters. Metagenomic binning was then performed using tetranucleotide frequency $\mathrm{ESOMs}^{[79]}$ and the ggkbase manual binning platform. 


\section{Sampling, DNA extraction, metagenomic sequencing and analyses for Tibet and Yunnan hot springs.}

Hot spring sediment samples were collected in 2016 from Tibet Plateau and Yunnan Province, China. The microbial community and structure in those samples have been reported previously (Chen et al. 2019) ${ }^{[41]}$. Samples were collected from the hot spring pools using a sterile iron spoon and stored in $50 \mathrm{ml}$ sterile plastic tubes. The tubes were transported using dry ice to the lab, and stored at $-80^{\circ} \mathrm{C}$ for further analyses and treatment including DNA extraction. The genomic DNA was extracted from the samples using FastDNA SPIN (MP Biomedicals, Irvine, CA) according to the manufacturer's instructions, and purified for library construction. The purified genomic DNA was subjected for metagenomic sequencing on an Illumina HiSeq2500 platform using paired-end $2 \times 150$ bp sequencing kit. The raw metagenomic reads were filtered to remove Illumina adapters, PhiX and other Illumina trace contaminants with BBTools Version 38.79, and low-quality bases and reads using Sickle (version 1.33; https://github.com/najoshi/sickle). The quality reads after filtering were assembled using metaSPAdes (version 3.10.1) with a kmer set of " $21,33,55,77,99,127$ ", and mapped to the corresponding assembled scaffolds using bowtie ${ }^{[55]}$ (version 2.3.5.1) for sequencing coverage calculation. The coverage of a given scaffold was calculated using the MetaBAT2 ${ }^{[56]}$ (version 2.12.1) script "jgi_summarize_bam_contig_depths". For each sample, the scaffolds $\geq 2500 \mathrm{bp}$ were binned using MetaBAT2 (version 2.12.1), with both tetranucleotide frequencies (TNF) and sequencing coverage of scaffolds considered. All the binned and unbinned scaffolds $\geq 1000 \mathrm{bp}$ were uploaded to ggKbase (http://ggkbase.berkeley.edu/) for manual curation of genome bins based on GC content, sequencing coverage and taxonomic information of each scaffold ${ }^{[57]}$. The ggKbase genome bins were curated individually to fix local assembly errors using ra2.py as previously described ${ }^{[58]}$.

\section{Ribosomal Phylogeny}

Genomes were searched for 16 ribosomal proteins ${ }^{[59]}$ using GOOSOS.py (https://github.com/jwestrob/GOOSOS) and the following HMMs: Ribosomal_L2 (K02886), Ribosomal_L3 (K02906), Ribosomal_L4 (K02926), Ribosomal_L5 (K02931), Ribosomal_L6 (K02933), Ribosomal_L14 (K02874), Ribosomal_L15 (K02876), Ribosomal_L16 (K02878), Ribosomal_L18 (K02881), Ribosomal_L22 (K02890), Ribosomal_L24 (K02895), Ribosomal_S3 (K02982), Ribosomal_S8 (K02994), Ribosomal_S10 (PF00338), Ribosomal_S17 (K02961), and Ribosomal_S19 (K02965). Ribosomal S10 model PF00338 was used in place of K02946 because the KOFAM model had a much lower hit rate than the other KOFAM models used.

Genomes containing at least 8 of these 16 proteins on a single scaffold were then used for further analysis. Retrieved protein sequences for each model were aligned individually using FAMSA $^{[90]}$ and concatenated using the script Concatenate_And_Align.py (https://github.com/jwestrob/GOOSOS/blob/master/Concatenate_And_Align.py). The concatenated alignment was trimmed using trimal ${ }^{[60]}$ with the parameter -gt 0.1 , keeping columns with fewer than $90 \%$ gaps. A guide tree was constructed using iQ-TREE $^{[61]}$ with the LG+FO+R10 model, and the final phylogeny was constructed using iQ-TREE with the 
LG+C20+FO model ${ }^{[63]}$ and 1000 ultrafast bootstrap replicates ${ }^{[62]}$; the number of mixture model components was capped at 20 due to computational constraints.

\section{Rubisco}

Rubisco large subunit sequences were identified using KOFAM model K01601 (rbcL) and PFAM model PF12338 ( $r b c S$ ). Phosphoribulokinase sequences were identified using the PFAM model PF00485 (PRK). Rubisco subtype classification was performed using a phylogeny estimated using Chloroflexi supergroup rbcL hits as well as reference sequences from Jaffe et ${\text { al. } 2019^{[64]} \text { as well as Banda et al. 2020 }}^{[13]}$. Phylogeny estimation was performed using iQ-TREE, using the LG+FO+G4 model as well as the ultrafast bootstrap approximation.

Sequences classified as Form I, Form II (outgroup), Form I', or Form I- $\alpha$ were then extracted from this dataset. Sequences corresponding to form $\mathrm{I}-\alpha$ were then searched against ggKbase using BLASTP ${ }^{[65]}$, and sequences with greater than $95 \%$ identity from unbinned metagenomic contigs were then added to the sequence dataset. These protein sequences, as well as the previously classified Form I, Form II, Form I', and Form I- $\alpha$ sequences, were then used to build the phylogeny displayed in Figure 3. This phylogeny was estimated using iQ-TREE with the LG+FO+G4 model as well as the ultrafast bootstrap approximation. Genome context diagrams were then generated using Clinker ${ }^{[66]}$.

\section{Hydrogenases}

Hydrogenase large subunit sequences were identified using the procedure outlined in Matheus-Carnevali et al. 2019 ${ }^{[67]}$ using custom HMMs (attached, supplemental). Chloroflexi supergroup genomes were searched using the NiFe group 123, NiFe group 4, and FeFe HMMs, then classification was performed using a phylogeny built with these HMM hits and references from Matheus-Carnevali et al. 2019 and $\mathrm{HydDB}^{[68]}$. Hydrogenase phylogenies were constructed using $\mathrm{iQ}-\mathrm{TREE}$ with the $\mathrm{LG}+\mathrm{FO}+\mathrm{R}$ models. Genome context diagrams in Figure 6 were generated using Clinker ${ }^{[66]}$.

Classification was then further refined by manual inspection of hydrogenase loci to ensure the presence of small subunit proteins as well as expected electron transfer and maturation machinery for each hydrogenase subtype.

The presence of genes encoding the catalytic subunit of hydrogenases was confirmed by phylogenetic analysis using references from Greening et al. ${ }^{[8]}$ (Fig. 6, Supp. Fig. S2, S3). Furthermore, visual inspection of hydrogenase gene clusters was performed and if at least the small subunit was not found in the vicinity of the large subunit, the genome was not included in hydrogenase counts. A combination of KOfam and Pfam annotations was used to determine the presence of any given gene cluster (Supplementary Table S3), although due to the less restrictive cutoffs for Pfam HMMs, the Pfam annotations were often used. Alternative annotations for the same genes were also taken into consideration in some cases (e.g., FeFe 
group $\mathrm{C}$ hydrogenases). The presence of a maturation protease in the vicinity of the hydrogenase genes, as well as multiple other hydrogenase expression/formation proteins, were considered as evidence of hydrogenase presence.

\section{Multiheme Cytochromes}

Proteins from Chloroflexi supergroup genomes were searched for $\mathrm{CxxCH}$ heme-binding motifs using a regular expression in Python. Proteins with more than 20 such motifs were classified as multiheme cytochromes for the purpose of this analysis.

\section{Sulfur Cycle}

Sulfur cycling genes were identified using hits to KOFAM HMMs corresponding to kegg modules M00176 (Assimilatory sulfate reduction), M00596 (Dissimilatory sulfate reduction) and M00595 (Thiosulfate oxidation). Models used to search for genes involved in DMSP metabolism include K07306 (dmsA), K00184/K07307 (dmsB), K00185/K07308 (dmsC), K16964 (ddhA), K16965 (ddhB), K16966 (ddhC), K16967 (dmoA), and K17285 (SELENBP1).

\section{CAZys}

Carbohydrate-Active enZymes (CAZys) were identified using dbCAN ${ }^{[69]}$ HMMs using an evalue threshold of 1e-15. CAZy counts were normalized by the size of the genome in mega-basepairs.

\section{CRISPR-Cas Systems}

CRISPR-Cas loci were identified and counted using CRISPRcasldentifier ${ }^{[70]}$ using default parameters. CRISPR repeats were identified using minced ${ }^{[87]}$.

\section{Identification of Genes Involved in the Nitrogen cycle}

Genomes were classified as having functional nitrogenase if those genomes contained hits to nitrogenase alpha (K02586) and beta (K02591) subunits as well as the catalytic subunit nifH (K02588). nxrAB loci were identified using the nxrAB HMMs provided in Anantharaman et al. 2016 ${ }^{[6]}$ using provided bitscore cutoffs, and scaffolds containing hits to both HMMs on the same scaffold were classified as $n x r A B . \quad \mathrm{N}_{2} \mathrm{O}$ reduction via norBC was searched for using KOFAM models K04561 and K02305, with only genomes containing hits to both HMMs considered valid. Other multi-gene systems searched for via this method are nirBD (K00362 and K00363), napAB (K02576 and K03568), nasAB (K00372 and K00360), and nrfAH (K03385 and K15876), which similarly required both HMMs to have hits in the same genome to identify a 
functional system. Other nitrogen gene markers used are nirK (K00368), nirS (K15864), nosZ (K00376), narB (K00367), and nirA (K00366).

The narGHIJ complex was detected using the KOFAM models narH/narY/nxrB (K00371) and narl/narV (K00374); the catalytic subunit, narG, lacks a KOFAM HMM model and the TIGRFAM narG HMM (from TIGRFAM, Karthik's Sulfur oxidation paper) was selected as an alternative model. Of those scaffolds containing the catalytic subunit narG, 73 had at least two of narH, narl, or narJ, and are considered complete for the purposes of this analysis. The presence of narGHIJ systems outside the clades in which it was detected by this analysis cannot be entirely discounted as systems with divergent narG sequences may exist that the TIGRFAM model does not capture with default cutoffs.

\section{Bacteriochlorophyll Biosynthesis Pathway Gene Identification}

Bacteriochlorophyll biosynthesis pathway genes were identified using KOFAMscan with provided bitscore cutoffs. Selected models include bchl (K03405), bchE (K04034), bchD (K03404), bchH (K03403), bchZ (K11335), bchC (K11337), bchK (K13605), bchX (K11333), bchY (K11334), bchG (K04040), bchP (K10960), bchN (K04038), bchB (K04039), bchL (K04037), bciC (K21058), bchF (K11336), bchJ (K04036), acsF (K04035), bchM (K03428), NYC1 (K13606), and fmoA/bacteriochlorophyll A protein (K08944). Where PFAM HMM models specific to a particular bacteriochlorophyll synthesis gene were available, the union of the corresponding PFAM and KOFAM HMMs were taken to represent hits to that particular gene, including bchJ (PF02830/V4R), bchL (PF02043/Bac_chlorC), bchF (PF07284/BCHF), fmoA/bacteriochlorphyll A protein (PF02327/BChl_A), and bchM (PF07109/Mg-por_mtran_C).

Chlorosome genes were defined as hits of greater than or equal to $40 \%$ identity to representative chlorosome genes from Chloroflexus aurantiacus available in uniprot (csmA, $\operatorname{csm} M, \operatorname{csm} N, \operatorname{csm} O)$.

Sequences for photosystem reaction center II subunits pufL and pufM within the Chloroflexi supergroup were obtained by searching with pfam model PF00124 (Photo_RC) and applying the model-designated GA cutoff; genomes with two hits, one for each subunit, were considered to have the pufLM complex. Proteins containing two domain-level hits to PF00124 and approximately twice the length of pufL were considered puf $L M$ fusion events.

\section{Reductive Dehalogenases}

Reductive dehalogenase enzymes were searched for using the PFAM model PF13486 (Dehalogenase) as well as KOFAM models K01560, K01563, and K01561. The count of reductive dehalogenase enzymes per genome (Fig. 2) is defined as the union of all such hits per genome. 


\section{Data Availability}

All supplementary data, including nucleotide and protein fasta files for each genome in the dataset and associated annotations, for this project is available at https://figshare.com/projects/Chloroflexi_Supergroup/120267.

\section{References}

[1] Pierson, B. K., and R. W. Castenholz. 1974. "A Phototrophic Gliding Filamentous Bacterium of Hot Springs, Chloroflexus Aurantiacus, Gen. and Sp. Nov." Archives of Microbiology 100 (1): 5-24.

[2] Yamada, Takeshi, and Yuji Sekiguchi. 2009. "Cultivation of Uncultured Chloroflexi Subphyla: Significance and Ecophysiology of Formerly Uncultured Chloroflexi 'Subphylum I' with Natural and Biotechnological Relevance." Microbes and Environments / JSME 24 (3): 205-16.

[3] Schoch CL, et al. NCBI Taxonomy: a comprehensive update on curation, resources and tools. Database (Oxford). 2020: baaa062.

[4] Diamond, Spencer, Peter F. Andeer, Zhou Li, Alexander Crits-Christoph, David Burstein, Karthik Anantharaman, Katherine R. Lane, et al. 2019. "Mediterranean Grassland Soil C-N Compound Turnover Is Dependent on Rainfall and Depth, and Is Mediated by Genomically Divergent Microorganisms." Nature Microbiology, May. https://doi.org/10.1038/s41564-019-0449-y.

[5] Woodcroft, Ben J., Caitlin M. Singleton, Joel A. Boyd, Paul N. Evans, Joanne B. Emerson, Ahmed A. F. Zayed, Robert D. Hoelzle, et al. 2018. "Genome-Centric View of Carbon Processing in Thawing Permafrost.” Nature 560 (7716): 49-54.

[6] Anantharaman, Karthik, Bela Hausmann, Sean P. Jungbluth, Rose S. Kantor, Adi Lavy, Lesley A. Warren, Michael S. Rappé, et al. 2018. "Expanded Diversity of Microbial Groups That Shape the Dissimilatory Sulfur Cycle." The ISME Journal 12 (7): 1715-28.

[7] Mehrshad, Maliheh, Michaela M. Salcher, Yusuke Okazaki, Shin-Ichi Nakano, Karel Šimek, Adrian-Stefan Andrei, and Rohit Ghai. 2018. "Hidden in Plain Sight-Highly Abundant and Diverse Planktonic Freshwater Chloroflexi." Microbiome 6 (1): 176.

[8] Hug, Laura A., Robert G. Beiko, Annette R. Rowe, Ruth E. Richardson, and Elizabeth A. Edwards. 2012. "Comparative Metagenomics of Three Dehalococcoides-Containing Enrichment Cultures: The Role of the Non-Dechlorinating Community." BMC Genomics 13 (July): 327. 
[9] Zheng, Yu, Ayana Saitou, Chiung-Mei Wang, Atsushi Toyoda, Yohei Minakuchi, Yuji Sekiguchi, Kenji Ueda, et al. 2019. "Genome Features and Secondary Metabolites Biosynthetic Potential of the Class Ktedonobacteria." Frontiers in Microbiology 10 (April): 893.

[10] Tsuji, J. M., N. A. Shaw, S. Nagashima, J. J. Venkiteswaran, S. L. Schiff, S. Hanada, M. Tank, and J. D. Neufeld. 2020. "Anoxygenic Phototrophic Chloroflexota Member Uses a Type I Reaction Center." bioRxiv. https://doi.org/10.1101/2020.07.07.190934.

[11] Ward, Lewis M., James Hemp, Patrick M. Shih, Shawn E. McGlynn, and Woodward W. Fischer. 2018. "Evolution of Phototrophy in the Chloroflexi Phylum Driven by Horizontal Gene Transfer." Frontiers in Microbiology 9 (February): 260.

[12] Ward, Lewis M., Usha F. Lingappa, John P. Grotzinger, and Woodward W. Fischer. 2020. "Microbial Mats in the Turks and Caicos Islands Reveal Diversity and Evolution of Phototrophy in the Chloroflexota Order Aggregatilineales." Environmental Microbiome 15 (1): 9.

[13] Banda, Douglas M., Jose H. Pereira, Albert K. Liu, Douglas J. Orr, Michal Hammel, Christine He, Martin A. J. Parry, et al. 2020. "Novel Bacterial Clade Reveals Origin of Form I Rubisco." Nature Plants 6 (9): 1158-66.

[14] King, C. E., and G. M. King. 2014. "Description of Thermogemmatispora Carboxidivorans Sp. Nov., a Carbon-Monoxide-Oxidizing Member of the Class Ktedonobacteria Isolated from a Geothermally Heated Biofilm, and Analysis of Carbon Monoxide Oxidation by Members of the Class Ktedonobacteria." International Journal of Systematic and Evolutionary Microbiology 64 (Pt 4): 1244-51.

[15] Yabe, Shuhei, Yasuteru Sakai, Keietsu Abe, and Akira Yokota. 2017. "Diversity of Ktedonobacteria with Actinomycetes-Like Morphology in Terrestrial Environments." Microbes and Environments / JSME 32 (1): 61-70.

[16] Islam, Zahra F., Paul R. F. Cordero, Joanna Feng, Ya-Jou Chen, Sean K. Bay, Thanavit Jirapanjawat, Roslyn M. Gleadow, et al. 2019. "Two Chloroflexi Classes Independently Evolved the Ability to Persist on Atmospheric Hydrogen and Carbon Monoxide." The ISME Journal 13 (7): 1801-13.

[17] Islam, Zahra F., Caitlin Welsh, Katherine Bayly, Rhys Grinter, Gordon Southam, Emma J. Gagen, and Chris Greening. 2020. "A Widely Distributed Hydrogenase Oxidises Atmospheric H2 during Bacterial Growth." The ISME Journal 14 (11): 2649-58.

[18] Parks, Donovan H., Maria Chuvochina, Pierre-Alain Chaumeil, Christian Rinke, Aaron J. Mussig, and Philip Hugenholtz. 2020. "A Complete Domain-to-Species Taxonomy for Bacteria and Archaea." Nature Biotechnology 38 (9): 1079-86. 
[19] Parks, Donovan H., Maria Chuvochina, David W. Waite, Christian Rinke, Adam Skarshewski, Pierre-Alain Chaumeil, and Philip Hugenholtz. 2018. "A Standardized Bacterial Taxonomy Based on Genome Phylogeny Substantially Revises the Tree of Life." Nature Biotechnology 36 (10): 996-1004.

[20] Ortiz, Maximiliano, Pok Man Leung, Guy Shelley, Marc W. Van Goethem, Sean K. Bay, Karen Jordaan, Surendra Vikram, et al. 2020. "A Genome Compendium Reveals Diverse Metabolic Adaptations of Antarctic Soil Microorganisms." bioRxiv. https://doi.org/10.1101/2020.08.06.239558.

[21] Engelberts, J. Pamela, Steven J. Robbins, Jasper M. de Goeij, Manuel Aranda, Sara C. Bell, and Nicole S. Webster. 2020. "Characterization of a Sponge Microbiome Using an Integrative Genome-Centric Approach.” The ISME Journal 14 (5): 1100-1110.

[22] Kube, Michael, Alfred Beck, Stephen H. Zinder, Heiner Kuhl, Richard Reinhardt, and Lorenz Adrian. 2005. "Genome Sequence of the Chlorinated Compound-Respiring Bacterium Dehalococcoides Species Strain CBDB1.” Nature Biotechnology 23 (10): 1269-73.

[23] Zinder, Stephen H. 2016. "The Genus Dehalococcoides." In Organohalide-Respiring Bacteria, edited by Lorenz Adrian and Frank E. Löffler, 107-36. Berlin, Heidelberg: Springer Berlin Heidelberg.

[24] Mehrshad, Maliheh, Francisco Rodriguez-Valera, Mohammad Ali Amoozegar, Purificación López-García, and Rohit Ghai. 2018. "The Enigmatic SAR202 Cluster up Close: Shedding Light on a Globally Distributed Dark Ocean Lineage Involved in Sulfur Cycling." The ISME Journal 12 (3): 655-68.

[25] Morris, R. M., M. S. Rappé, E. Urbach, S. A. Connon, and S. J. Giovannoni. 2004. "Prevalence of the Chloroflexi-Related SAR202 Bacterioplankton Cluster throughout the Mesopelagic Zone and Deep Ocean." Applied and Environmental Microbiology 70 (5): 2836-42.

[26] Hug, Laura A., Cindy J. Castelle, Kelly C. Wrighton, Brian C. Thomas, Itai Sharon, Kyle R. Frischkorn, Kenneth H. Williams, Susannah G. Tringe, and Jillian F. Banfield. 2013. "Community Genomic Analyses Constrain the Distribution of Metabolic Traits across the Chloroflexi Phylum and Indicate Roles in Sediment Carbon Cycling." Microbiome 1 (1): 22.

[27] Psencík, Jakub, Aaron M. Collins, Lassi Liljeroos, Mika Torkkeli, Pasi Laurinmäki, Hermanus M. Ansink, Teemu P. Ikonen, et al. 2009. "Structure of Chlorosomes from the Green Filamentous Bacterium Chloroflexus Aurantiacus.” Journal of Bacteriology 191 (21): 6701-8.

[28] Thauer, Rudolf K., Anne-Kristin Kaster, Henning Seedorf, Wolfgang Buckel, and Reiner Hedderich. 2008. "Methanogenic Archaea: Ecologically Relevant Differences in Energy Conservation." Nature Reviews. Microbiology 6 (8): 579-91. 
[29] Wagner, Tristan, Jürgen Koch, Ulrich Ermler, and Seigo Shima. 2017. "Methanogenic Heterodisulfide Reductase (HdrABC-MvhAGD) Uses Two Noncubane [4Fe-4S] Clusters for Reduction." Science 357 (6352): 699-703.

[30] Schäfer, Caspar, Martin Bommer, Sandra E. Hennig, Jae-Hun Jeoung, Holger Dobbek, and Oliver Lenz. 2016. "Structure of an Actinobacterial-Type [NiFe]-Hydrogenase Reveals Insight into O2-Tolerant H2 Oxidation.” Structure 24 (2): 285-92.

[31] Leu, Andy O., Chen Cai, Simon J. Mcllroy, Gordon Southam, Victoria J. Orphan, Zhiguo Yuan, Shihu Hu, and Gene W. Tyson. 2020. "Anaerobic Methane Oxidation Coupled to Manganese Reduction by Members of the Methanoperedenaceae." The ISME Journal 14 (4): 1030-41.

[32] Edwards, Marcus J., David J. Richardson, Catarina M. Paquete, and Thomas A. Clarke. 2020. "Role of Multiheme Cytochromes Involved in Extracellular Anaerobic Respiration in Bacteria." Protein Science: A Publication of the Protein Society 29 (4): 830-42.

[33] Vigneron, Adrien, Perrine Cruaud, Alexander I. Culley, Raoul-Marie Couture, Connie Lovejoy, and Warwick F. Vincent. 2020. "Sulfur Intermediates as New Biogeochemical Hubs in an Aquatic Model Microbial Ecosystem." Research Square. https://doi.org/10.21203/rs.3.rs-32029/v1.

[34] Robbins, S. J., W. Song, J. P. Engelberts, B. Glasl, B. M. Slaby, J. Boyd, E. Marangon, et al. 2021. "A Genomic View of the Microbiome of Coral Reef Demosponges." The ISME Journal 15 (6): 1641-54.

[35] Anantharaman, Karthik, Christopher T. Brown, Laura A. Hug, Itai Sharon, Cindy J. Castelle, Alexander J. Probst, Brian C. Thomas, et al. 2016. "Thousands of Microbial Genomes Shed Light on Interconnected Biogeochemical Processes in an Aquifer System." Nature Communications 7 (October): 13219.

[36] Davis, Kathryn E. R., Parveen Sangwan, and Peter H. Janssen. 2011. "Acidobacteria, Rubrobacteridae and Chloroflexi Are Abundant among Very Slow-Growing and Mini-Colony-Forming Soil Bacteria." Environmental Microbiology 13 (3): 798-805.

[37] Zhou, Zhichao, Yang Liu, Wei Xu, Jie Pan, Zhu-Hua Luo, and Meng Li. 2020. "Genomeand Community-Level Interaction Insights into Carbon Utilization and Element Cycling Functions of Hydrothermarchaeota in Hydrothermal Sediment." mSystems 5 (1). https://doi.org/10.1128/mSystems.00795-19.

[38] He, Christine, Ray Keren, Michael L. Whittaker, Ibrahim F. Farag, Jennifer A. Doudna, Jamie H. D. Cate, and Jillian F. Banfield. 2021. "Genome-Resolved Metagenomics Reveals 
Site-Specific Diversity of Episymbiotic CPR Bacteria and DPANN Archaea in Groundwater Ecosystems." Nature Microbiology 6 (3): 354-65.

[39] Hernsdorf, Alex W., Yuki Amano, Kazuya Miyakawa, Kotaro Ise, Yohey Suzuki, Karthik Anantharaman, Alexander Probst, David Burstein, Brian C. Thomas, and Jillian F. Banfield. 2017. "Potential for Microbial H2 and Metal Transformations Associated with Novel Bacteria and Archaea in Deep Terrestrial Subsurface Sediments." The ISME Journal 11 (8): 1915-29.

[40] Waterworth, Samantha C., Eric W. Isemonger, Evan R. Rees, Rosemary A. Dorrington, and Jason C. Kwan. 2020. "Conserved Bacterial Genomes from Two Geographically Distinct Peritidal Stromatolite Formations Shed Light on Potential Functional Guilds." bioRxiv. https://doi.org/10.1101/818625.

[41] Chen, Lin-Xing, Basem Al-Shayeb, Raphaël Méheust, Wen-Jun Li, Jennifer A. Doudna, and Jillian F. Banfield. 2019. "Candidate Phyla Radiation Roizmanbacteria From Hot Springs Have Novel and Unexpectedly Abundant CRISPR-Cas Systems." Frontiers in Microbiology 10 (May): 928.

[42] Nierychlo, Marta, Aleksandra Milobedzka, Francesca Petriglieri, Bianca Mcllroy, Per Halkjær Nielsen, and Simon Jon Mcllroy. 2019. "The Morphology and Metabolic Potential of the Chloroflexi in Full-Scale Activated Sludge Wastewater Treatment Plants." FEMS Microbiology Ecology 95 (2). https://doi.org/10.1093/femsec/fiy228.

[43] Shih, Patrick M., Lewis M. Ward, and Woodward W. Fischer. 2017. "Evolution of the 3-Hydroxypropionate Bicycle and Recent Transfer of Anoxygenic Photosynthesis into the Chloroflexi." Proceedings of the National Academy of Sciences of the United States of America 114 (40): 10749-54.

[44] Rissanen, Antti J., Taija Saarela, Helena Jäntti, Moritz Buck, Sari Peura, Sanni L. Aalto, Anne Ojala, et al. 2021. "Vertical Stratification Patterns of Methanotrophs and Their Genetic Controllers in Water Columns of Oxygen-Stratified Boreal Lakes." FEMS Microbiology Ecology 97 (2). https://doi.org/10.1093/femsec/fiaa252.

[45] Crits-Christoph, Alexander, Spencer Diamond, Cristina N. Butterfield, Brian C. Thomas, and Jillian F. Banfield. 2018. "Novel Soil Bacteria Possess Diverse Genes for Secondary Metabolite Biosynthesis." Nature 558 (7710): 440-44.

[46] Sharrar, Allison M., Alexander Crits-Christoph, Raphaël Méheust, Spencer Diamond, Evan P. Starr, and Jillian F. Banfield. 2020. "Bacterial Secondary Metabolite Biosynthetic Potential in Soil Varies with Phylum, Depth, and Vegetation Type." mBio 11 (3). https://doi.org/10.1128/mBio.00416-20. 
[47] Clark, Karen, Ilene Karsch-Mizrachi, David J. Lipman, James Ostell, and Eric W. Sayers. 2016. "GenBank." Nucleic Acids Research 44 (D1): D67-72.

[48] Davis JJ, Wattam AR, Aziz RK, Brettin T, Butler R, Butler RM, Chlenski P, Conrad N, Dickerman A, Dietrich EM, Gabbard JL, Gerdes S, Guard A, Kenyon RW, Machi D, Mao C, Murphy-Olson D, Nguyen M, Nordberg EK, Olsen GJ, Olson RD, Overbeek JC, Overbeek R, Parrello B, Pusch GD, Shukla M, Thomas C, VanOeffelen M, Vonstein V, Warren AS, Xia F, Xie D, Yoo H, Stevens R. The PATRIC Bioinformatics Resource Center: expanding data and analysis capabilities. Nucleic Acids Res. 2020 Jan 8;48(D1):D606-D612. doi: 10.1093/nar/gkz943. PMID: 31667520. PMCID: PMC7145515.

[49] Olm, Matthew R., Christopher T. Brown, Brandon Brooks, and Jillian F. Banfield. 2017. "dRep: A Tool for Fast and Accurate Genomic Comparisons That Enables Improved Genome Recovery from Metagenomes through de-Replication." The ISME Journal 11 (12): 2864-68.

[50] Parks, Donovan H., Michael Imelfort, Connor T. Skennerton, Philip Hugenholtz, and Gene W. Tyson. 2015. "CheckM: Assessing the Quality of Microbial Genomes Recovered from Isolates, Single Cells, and Metagenomes.” Genome Research 25 (7): 1043-55.

[51] Aramaki, Takuya, Romain Blanc-Mathieu, Hisashi Endo, Koichi Ohkubo, Minoru Kanehisa, Susumu Goto, and Hiroyuki Ogata. 2020. "KofamKOALA: KEGG Ortholog Assignment Based on Profile HMM and Adaptive Score Threshold." Bioinformatics 36 (7): 2251-52.

[52] Legendre, Pierre, and Eugene D. Gallagher. 2001. "Ecologically Meaningful Transformations for Ordination of Species Data." Oecologia 129 (2): 271-80.

[53] McInnes, Leland, John Healy, and James Melville. 2018. "UMAP: Uniform Manifold Approximation and Projection for Dimension Reduction." arXiv [stat.ML]. arXiv. http://arxiv.org/abs/1802.03426.

[54] Letunic I and Bork P (2021) Nucleic Acids Res doi: 10.1093/nar/gkab301 Interactive Tree Of Life (iTOL) v5: an online tool for phylogenetic tree display and annotation

[55] Langmead, Ben, and Steven L. Salzberg. 2012. "Fast Gapped-Read Alignment with Bowtie 2." Nature Methods 9 (4): 357-59.

[56] Kang, Dongwan D., Feng Li, Edward Kirton, Ashleigh Thomas, Rob Egan, Hong An, and Zhong Wang. 2019. "MetaBAT 2: An Adaptive Binning Algorithm for Robust and Efficient Genome Reconstruction from Metagenome Assemblies.” PeerJ 7 (July): e7359.

[57] Chen, Lin-Xing, Karthik Anantharaman, Alon Shaiber, A. Murat Eren, and Jillian F. Banfield. 2020. "Accurate and Complete Genomes from Metagenomes." Genome Research 30 (3): 315-33. 
[58] Brown, C. T., Hug, L. A., Thomas, B. C., Sharon, I., Castelle, C. J., Singh, A., et al. (2015). Unusual biology across a group comprising more than $15 \%$ of domain Bacteria. Nature 523, 208-211. doi: 10.1038/nature14486

[59] Hug, Laura A., Brett J. Baker, Karthik Anantharaman, Christopher T. Brown, Alexander J. Probst, Cindy J. Castelle, Cristina N. Butterfield, et al. 2016. "A New View of the Tree of Life." Nature Microbiology 1 (April): 16048.

[60] Capella-Gutiérrez, Salvador, José M. Silla-Martínez, and Toni Gabaldón. 2009. "trimAl: A Tool for Automated Alignment Trimming in Large-Scale Phylogenetic Analyses." Bioinformatics 25 (15): 1972-73.

[61] B.Q. Minh, H.A. Schmidt, O. Chernomor, D. Schrempf, M.D. Woodhams, A. von Haeseler, R. Lanfear (2020) IQ-TREE 2: New models and efficient methods for phylogenetic inference in the genomic era. Mol. Biol. Evol., 37:1530-1534. https://doi.org/10.1093/molbev/msaa015

[62] D.T. Hoang, O. Chernomor, A. von Haeseler, B.Q. Minh, L.S. Vinh (2018) UFBoot2: Improving the ultrafast bootstrap approximation. Mol. Biol. Evol., 35:518-522. https://doi.org/10.1093/molbev/msx281

[63] H.C. Wang, B.Q. Minh, S. Susko, A.J. Roger (2018) Modeling site heterogeneity with posterior mean site frequency profiles accelerates accurate phylogenomic estimation. Syst. Biol., 67:216-235. https://doi.org/10.1093/sysbio/syx068

[64] Jaffe, Alexander L., Cindy J. Castelle, Christopher L. Dupont, and Jillian F. Banfield. 2019. "Lateral Gene Transfer Shapes the Distribution of RuBisCO among Candidate Phyla Radiation Bacteria and DPANN Archaea." Molecular Biology and Evolution 36 (3): 435-46.

[65] Camacho C., Coulouris G., Avagyan V., Ma N., Papadopoulos J., Bealer K., \& Madden T.L. (2008) "BLAST+: architecture and applications." BMC Bioinformatics 10:421.

[66] clinker \& clustermap.js: Automatic generation of gene cluster comparison figures.

Gilchrist, C.L.M., Chooi, Y.-H., 2020.

Bioinformatics. doi: https://doi.org/10.1093/bioinformatics/btab007

[67] Matheus Carnevali, Paula B., Frederik Schulz, Cindy J. Castelle, Rose S. Kantor, Patrick M. Shih, Itai Sharon, Joanne M. Santini, et al. 2019. "Hydrogen-Based Metabolism as an Ancestral Trait in Lineages Sibling to the Cyanobacteria." Nature Communications 10 (1): 463.

[68] Søndergaard, Dan, Christian N. S. Pedersen, and Chris Greening. 2016. "HydDB: A Web Tool for Hydrogenase Classification and Analysis." Scientific Reports 6 (September): 34212. 
[69] Yin, Yanbin, Xizeng Mao, Jincai Yang, Xin Chen, Fenglou Mao, and Ying Xu. 2012. "dbCAN: A Web Resource for Automated Carbohydrate-Active Enzyme Annotation." Nucleic Acids Research 40 (Web Server issue): W445-51.

[70] Padilha, Victor A., Omer S. Alkhnbashi, Shiraz A. Shah, André C. P. L. F. de Carvalho, and Rolf Backofen. 2020. "CRISPRcasldentifier: Machine Learning for Accurate Identification and Classification of CRISPR-Cas Systems.” GigaScience $9 \quad$ (6). https://doi.org/10.1093/gigascience/giaa062.

[71] Mistry, Jaina, Sara Chuguransky, Lowri Williams, Matloob Qureshi, Gustavo A. Salazar, Erik L. L. Sonnhammer, Silvio C. E. Tosatto, et al. 2021. "Pfam: The Protein Families Database in 2021." Nucleic Acids Research 49 (D1): D412-19.

[72] McKinney, W. \& others, 2010. Data structures for statistical computing in python. In Proceedings of the 9th Python in Science Conference. pp. 51-56.

[73] Harris, C.R. et al., 2020. Array programming with NumPy. Nature, 585, pp.357-362.

[74] Hunter, J.D., 2007. Matplotlib: A 2D graphics environment. Computing in science \&amp; engineering, 9(3), pp.90-95.

[75] Waskom, M. et al., 2017. mwaskom/seaborn: v0.8.1 (September 2017), Zenodo. Available at: https://doi.org/10.5281/zenodo.883859.

[76] Cock, P.J. et al., 2009. Biopython: freely available Python tools for computational molecular biology and bioinformatics. Bioinformatics, 25(11), pp.1422-1423.

[77] O. Tange (2018): GNU Parallel 2018, March 2018, https://doi.org/10.5281/zenodo.1146014.

[78] Lee, Patrick K. H., Dan Cheng, Ping Hu, Kimberlee A. West, Gregory J. Dick, Eoin L. Brodie, Gary L. Andersen, Stephen H. Zinder, Jianzhong He, and Lisa Alvarez-Cohen. 2011. "Comparative Genomics of Two Newly Isolated Dehalococcoides Strains and an Enrichment Using a Genus Microarray." The ISME Journal 5 (6): 1014-24.

[78] Mao, Xinwei, Benoit Stenuit, Julien Tremblay, Ke Yu, Susannah G. Tringe, and Lisa Alvarez-Cohen. 2019. "Structural Dynamics and Transcriptomic Analysis of Dehalococcoides Mccartyi within a TCE-Dechlorinating Community in a Completely Mixed Flow Reactor." Water Research 158 (July): 146-56.

[79] Dick, Gregory J., Anders F. Andersson, Brett J. Baker, Sheri L. Simmons, Brian C. Thomas, A. Pepper Yelton, and Jillian F. Banfield. 2009. "Community-Wide Analysis of Microbial Genome Sequence Signatures.” Genome Biology 10 (8): R85. 
[80] Peng, Yu, Henry C. M. Leung, S. M. Yiu, and Francis Y. L. Chin. 2012. "IDBA-UD: A de Novo Assembler for Single-Cell and Metagenomic Sequencing Data with Highly Uneven Depth." Bioinformatics 28 (11): 1420-28.

[81] Sieber, Christian M. K., Blair G. Paul, Cindy J. Castelle, Ping Hu, Susannah G. Tringe, David L. Valentine, Gary L. Andersen, and Jillian F. Banfield. 2019. "Unusual Metabolism and Hypervariation in the Genome of a Gracilibacterium (BD1-5) from an Oil-Degrading Community." mBio 10 (6). https://doi.org/10.1128/mBio.02128-19.

[82] Matheus Carnevali, Paula B., Adi Lavy, Alex D. Thomas, Alexander Crits-Christoph, Spencer Diamond, Raphaël Méheust, Matthew R. Olm, et al. 2021. "Meanders as a Scaling Motif for Understanding of Floodplain Soil Microbiome and Biogeochemical Potential at the Watershed Scale." Microbiome 9 (1): 121.

[83] Hyatt, Doug, Gwo-Liang Chen, Philip F. Locascio, Miriam L. Land, Frank W. Larimer, and Loren J. Hauser. 2010. "Prodigal: Prokaryotic Gene Recognition and Translation Initiation Site Identification." BMC Bioinformatics 11 (March): 119.

[84] Westcott, Sarah L., and Patrick D. Schloss. 2015. "De Novo Clustering Methods Outperform Reference-Based Methods for Assigning 16S rRNA Gene Sequences to Operational Taxonomic Units." PeerJ 3 (December): e1487.

[85] UniProt Consortium. 2019. "UniProt: A Worldwide Hub of Protein Knowledge." Nucleic Acids Research 47 (D1): D506-15.

[86] Kanehisa, M., and S. Goto. 2000. "KEGG: Kyoto Encyclopedia of Genes and Genomes." Nucleic Acids Research 28 (1): 27-30.

[87] Minced: Mining CRISPRs in Environmental Datasets [https://github.com/ctSkennerton/minced/tree/master]

[88] Assfalg, Michael, Ivano Bertini, Mireille Bruschi, Caroline Michel, and Paola Turano. 2002. "The Metal Reductase Activity of Some Multiheme Cytochromes c: NMR Structural Characterization of the Reduction of chromium(VI) to chromium(III) by Cytochrome c(7)." Proceedings of the National Academy of Sciences of the United States of America 99 (15): 9750-54.

[89] Xu, Shuai, Alexandre Barrozo, Leonard M. Tender, Anna I. Krylov, and Mohamed Y. El-Naggar. 2018. "Multiheme Cytochrome Mediated Redox Conduction through Shewanella Oneidensis MR-1 Cells." Journal of the American Chemical Society 140 (32): 10085-89. 
[90] Deorowicz, Sebastian, Agnieszka Debudaj-Grabysz, and Adam Gudyś. 2016. "FAMSA: Fast and Accurate Multiple Sequence Alignment of Huge Protein Families." Scientific Reports 6 (September): 33964.

[91] Wu, Yu-Wei, Blake A. Simmons, and Steven W. Singer. 2016. "MaxBin 2.0: An Automated Binning Algorithm to Recover Genomes from Multiple Metagenomic Datasets." Bioinformatics 32 (4): 605-7.

[92] Johannes Alneberg, Brynjar Smári Bjarnason, Ino de Bruijn, Melanie Schirmer, Joshua Quick, Umer Z ljaz, Leo Lahti, Nicholas J Loman, Anders F Andersson \& Christopher Quince. 2014. Binning metagenomic contigs by coverage and composition. Nature Methods, doi: 10.1038/nmeth.3103

[93] Sieber, Christian M. K., Alexander J. Probst, Allison Sharrar, Brian C. Thomas, Matthias Hess, Susannah G. Tringe, and Jillian F. Banfield. 2018. "Recovery of Genomes from Metagenomes via a Dereplication, Aggregation and Scoring Strategy." Nature Microbiology 3 (7): 836-43. 


\section{Figures and Tables}
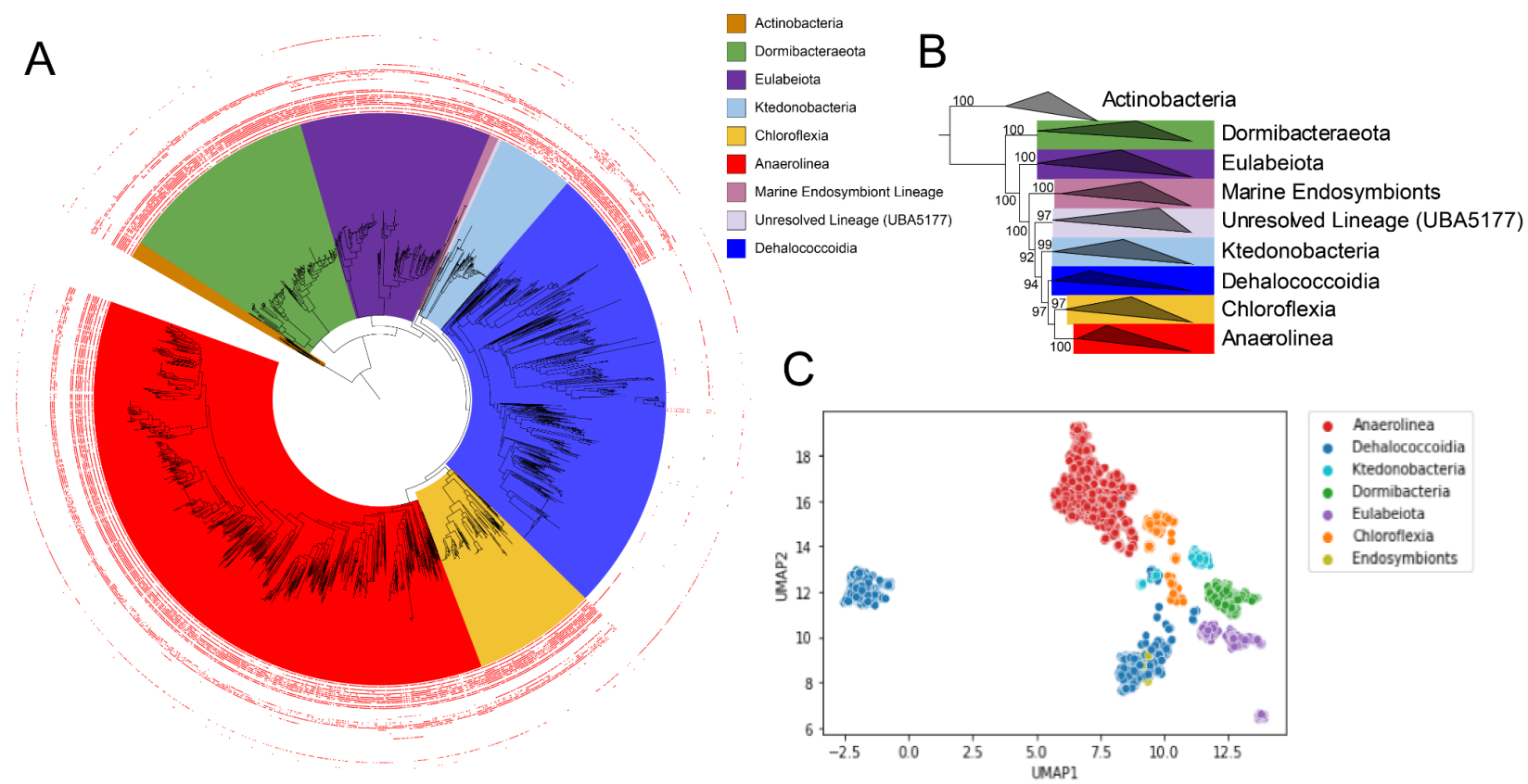

Figure 1. (A) Phylogenetic tree estimated using the PMSF C20 mixture model from concatenated sequences of 16 ribosomal proteins. Shown are the Chloroflexi supergroup, including the candidate phyla Dormibacteraeota and Eulabeiota, and the Actinobacteria as an outgroup. Red decorations along the outer ring indicate hits to KOFAM HMMs corresponding to the peptidoglycan biosynthesis pathway (map00550). (B) Rectangular view of the same tree showing the relative positions of the major subdivisions within the Chloroflexi supergroup as well as ultrafast bootstrap values for the deeply branching nodes which separate the groups. (C) UMAP embedding of a counts matrix representing KOFAM HMM hits across the dataset with taxonomic groups highlighted. Data was Hellinger normalized prior to projection with UMAP. 


\begin{tabular}{|l|l|}
\hline Phyletic Group & Number of Genomes \\
\hline Anaerolinea & 773 \\
\hline Dehalococcoidia & 551 \\
\hline Candidate phylum Dormibacteraeota & 241 \\
\hline Candidate phylum Eulabeiota & 229 \\
\hline Chloroflexia & 148 \\
\hline Ktedonobacteria & 92 \\
\hline Marine Endosymbiont Lineage & 11 \\
\hline Unresolved lineage (UB5177) & 3 \\
\hline
\end{tabular}

Table 1. Identified clades within the Chloroflexi supergroup, including candidate phyla Dormibacteraeota and Eulabeiota, and the number of identified genomes within each clade. 


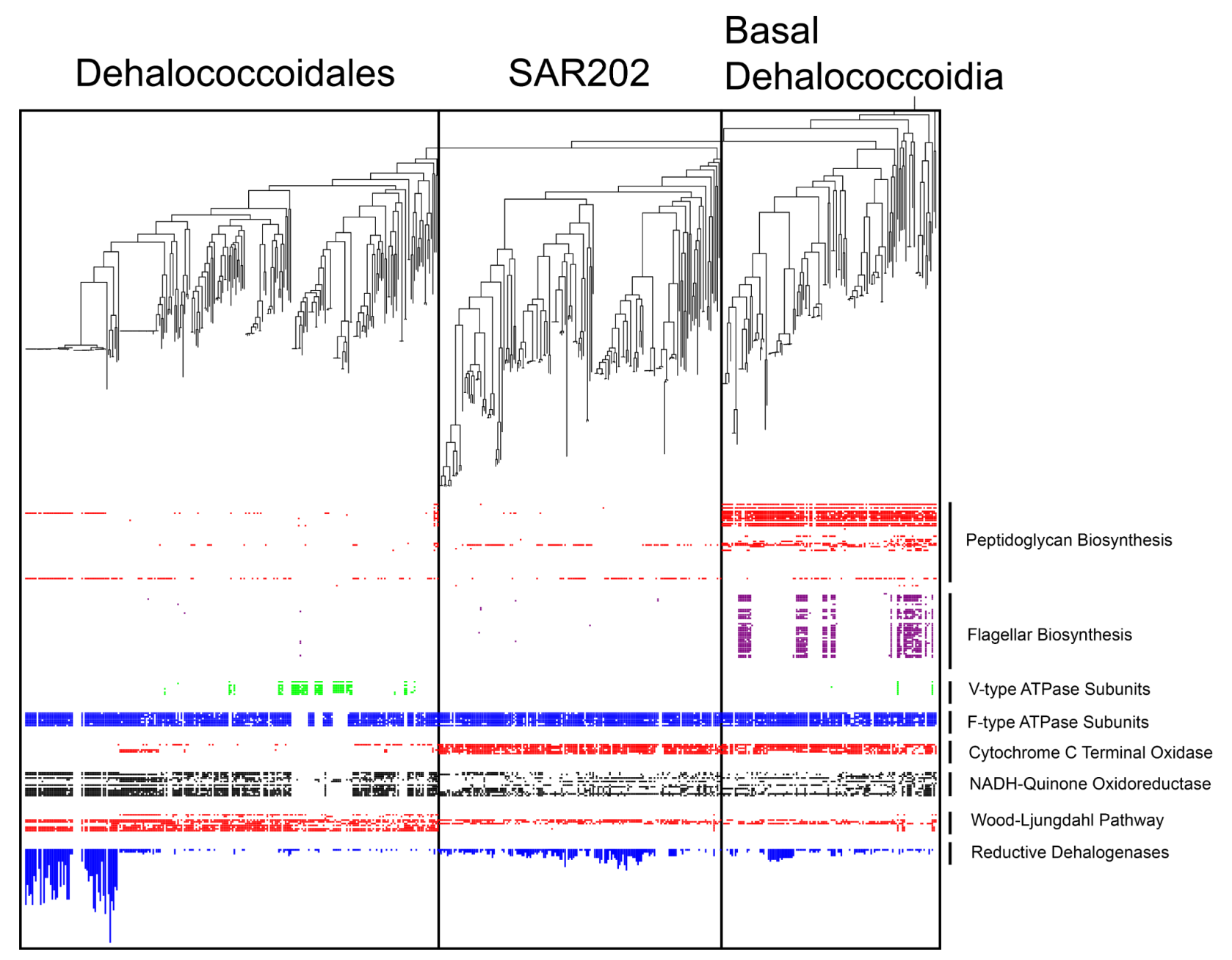

Figure 2. Subsection of the ribosomal phylogeny displayed in figure 1 focusing on the Dehalococcoidia, showing the three major functional subdivisions. The Basal Dehalococcoidia lineages are distinguished primarily by their intact peptidoglycan biosynthesis pathways and widely distributed flagellar biosynthesis capacities; the SAR202 lineages lack peptidoglycan biosynthesis capacity but retain many genes required for aerobic metabolism; and the Dehalococcoidales lineages lack markers for aerobic metabolism and contain representatives with high copy numbers of reductive dehalogenases such as the genus Dehalococcoides and its close relatives. 


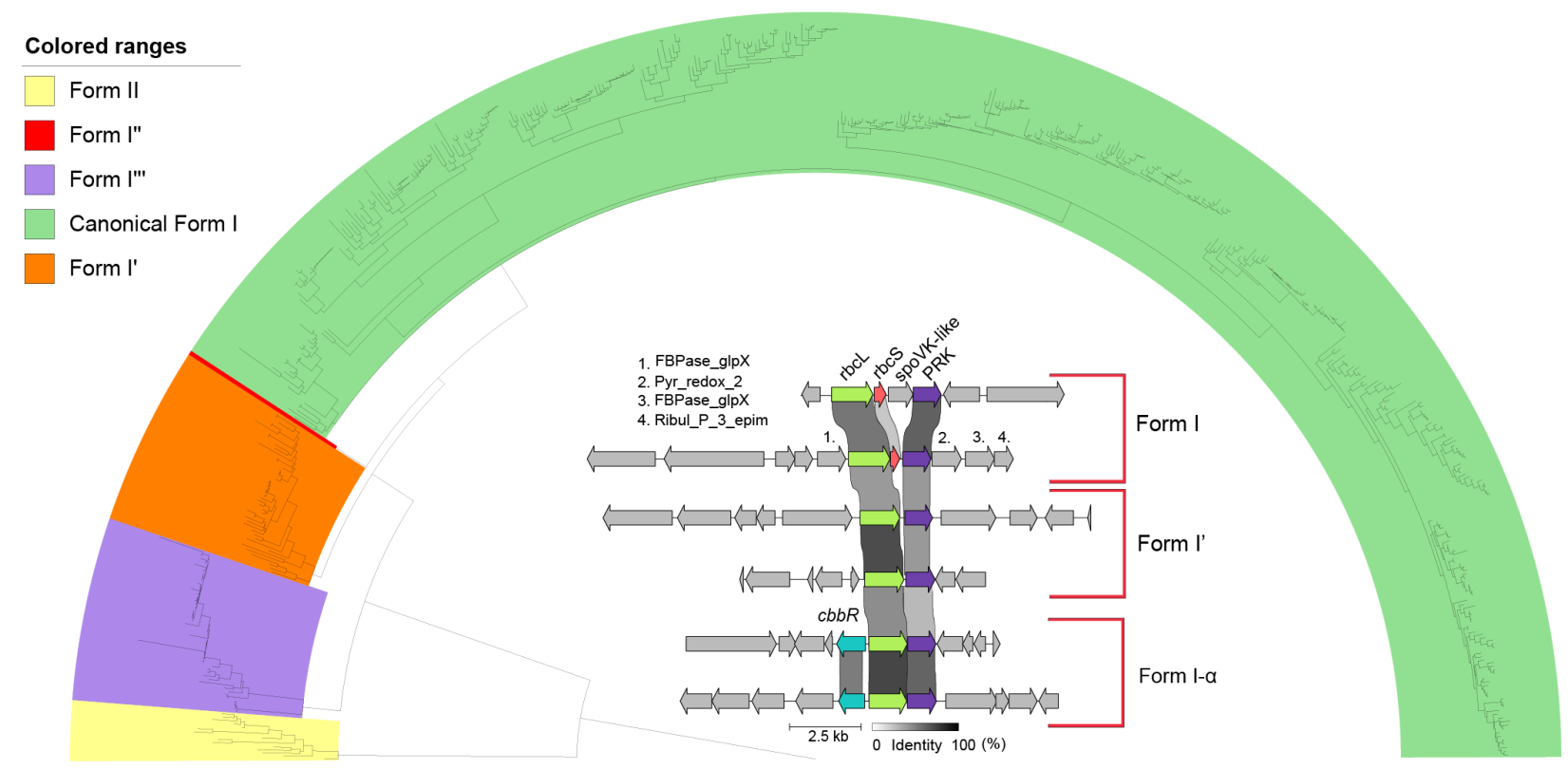

Fig. 3: Phylogenetic tree of rbcL forms I, I', I- $\alpha$, with form II used as an outgroup. Interior shows genomic context diagrams for gene clusters containing rbcL sequences corresponding to forms I, I', and 1- $\alpha$, highlighting the presence of PRK in all clusters and lack of $r b c S$ in clusters containing $r b c L$ sequences of forms I' and I- $\alpha$. 


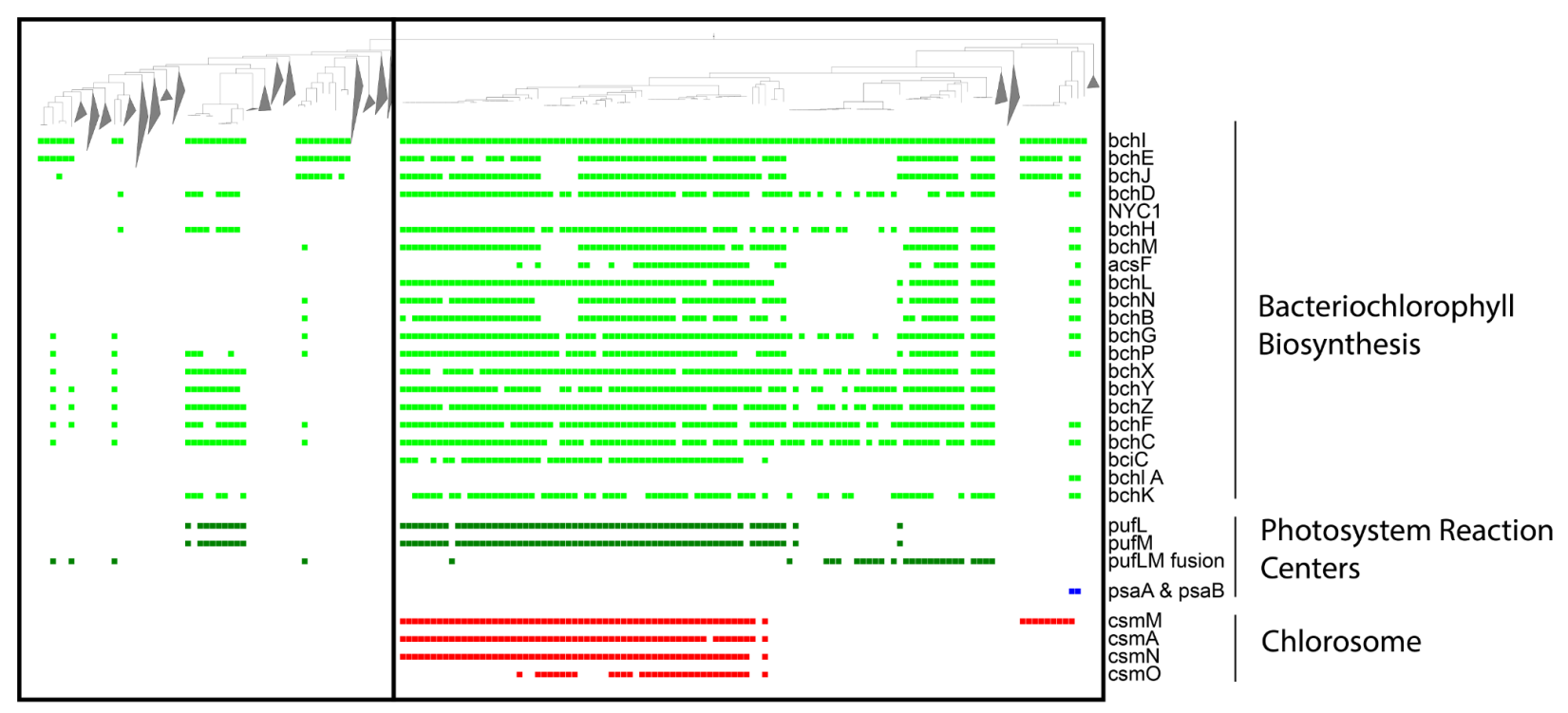

Anaerolinea

Chloroflexia

Figure 4. Subsection of the ribosomal phylogeny displayed in Figure 1 highlighting the groups Anaerolinea and Chloroflexia, which contain phototrophic representatives. Decorations indicate bacteriochlorophyll biosynthesis pathways (light green), photosystem II reaction centers (dark green), photosystem I reaction centers (dark blue), and chlorosome proteins (red). 


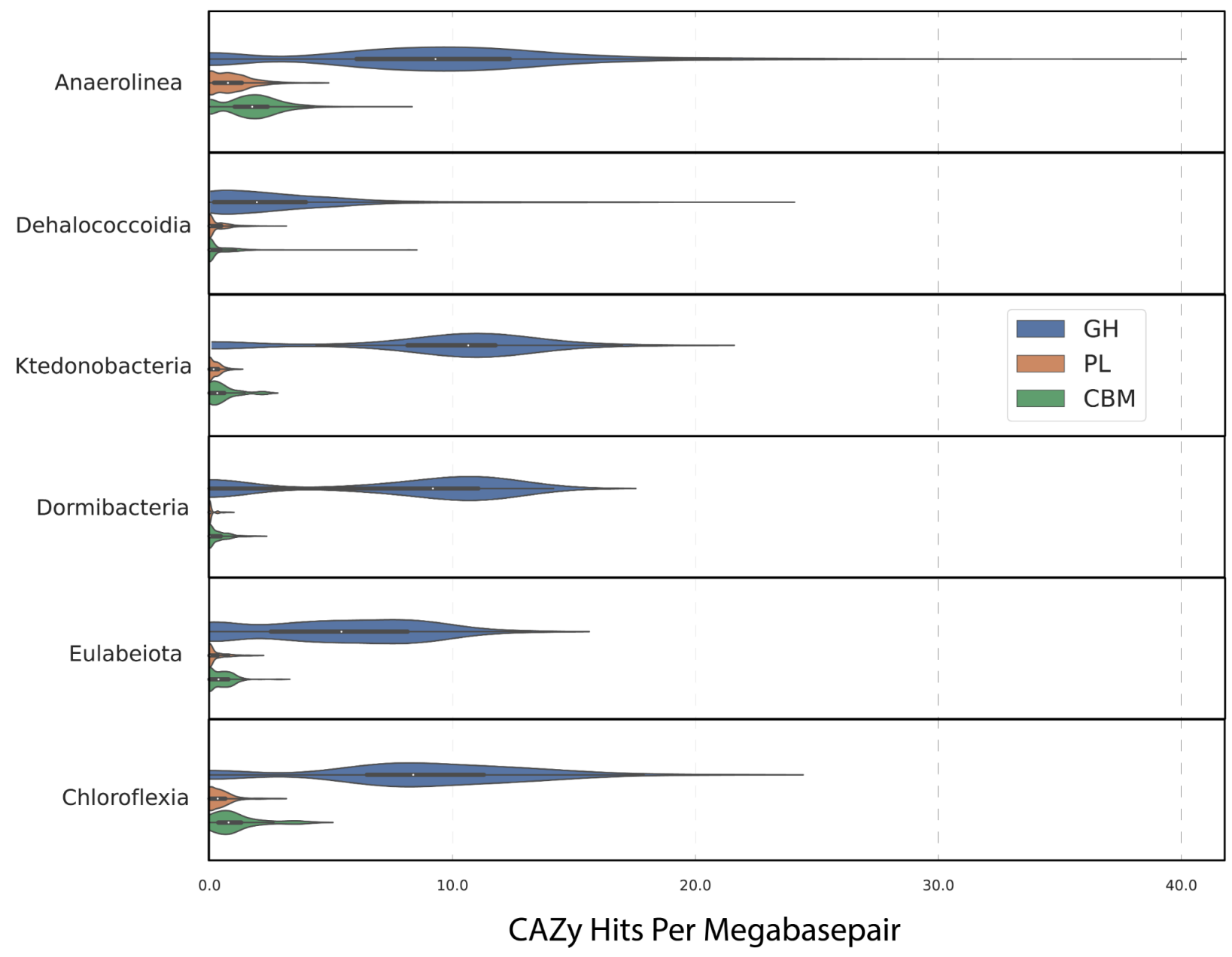

Figure 5. Distribution of CAZy copy number per genome normalized to genome length in megabasepairs for each major clade in the Chloroflexi supergroup. CAZy subtypes shown are Glycoside Hydrolase (GH), Polysaccharide Lyase (PL), and Carbohydrate-Binding Modules (CBM). 


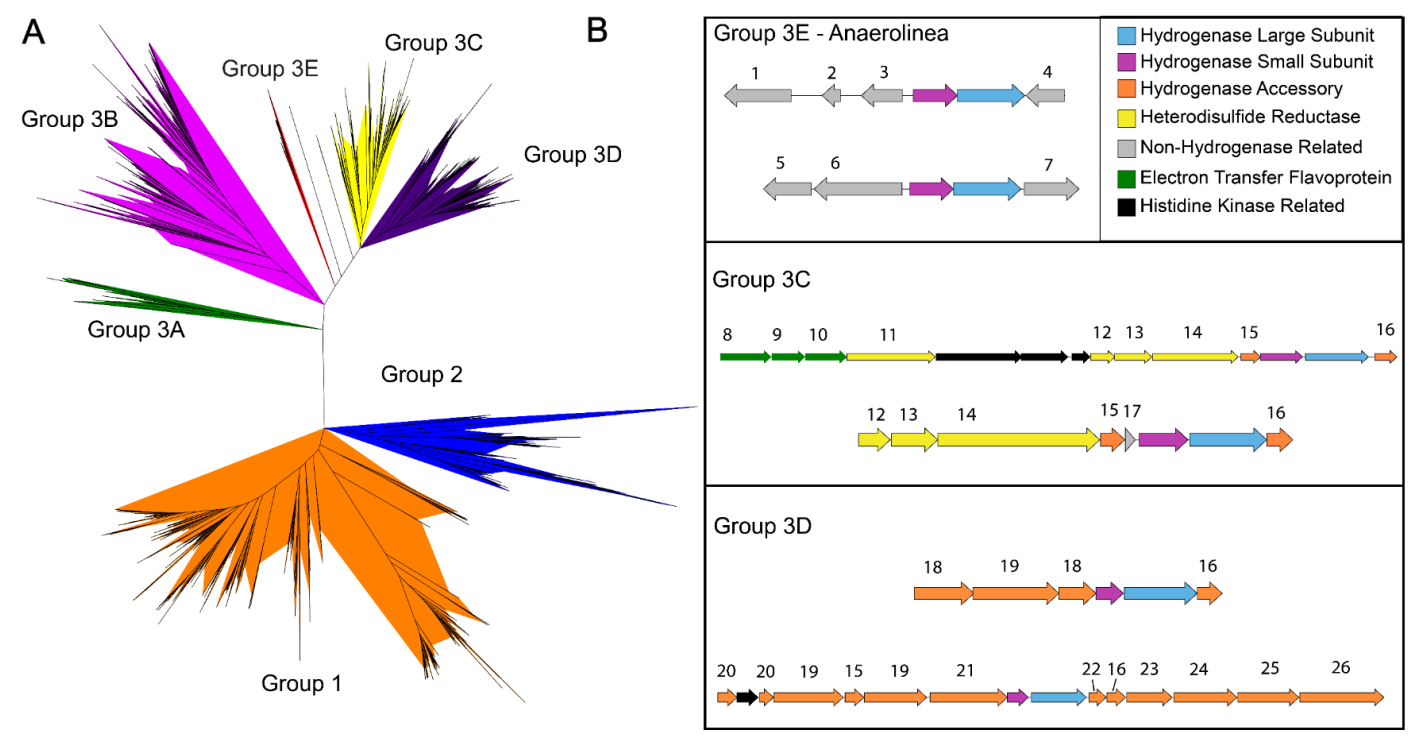

Figure 6. (A) Phylogeny of NiFe hydrogenase large subunit proteins from groups 1, 2, and 3, containing sequences from the Chloroflexi supergroup alongside references, and showing the relative placement of the newly proposed group 3E. (B) Genomic context diagrams for groups $3 \mathrm{E}, 3 \mathrm{C}$, and $3 \mathrm{D}$ showing the diversity in function between the NiFe hydrogenase subtypes in the Chloroflexi supergroup. Group 3E was not found to regularly co-occur with accessory proteins, whereas group $3 \mathrm{C}$ often occurred proximal to heterodisulfide reductase genes (hdrA2B2C2), and group 3D often co-occurred with numerous accessory and electron transfer subunit genes. Numbered genes are annotated as follows:

1. Serpin B, 2. FGE-Sulfatase, 3. Myo-inositol-1 monophosphatase, 4.

ADP-ribosylglycohydrolase, 5. UDP-Glucose-4-epimerase, 5. Ubiquinone biosynthesis protein, 6. Peptidase M23, 7. ETF-QO (electron acceptor subunit), 8. ETF Beta subunit, 9. ETF Alpha subunit, 10. Heterodisulfide reductase subunit D, 11. Heterodisulfide reductase subunit C2, 12. Heterodisulfide reductase subunit B2, 13. Heterodisulfide reductase subunit A2, 14. Hydrogenase Fe-S subunit, 15. Hycl protease, 16. PIN domain protein, 17. Bidirectional [NiFe] hydrogenase diaphorase subunit, 18. nqoF-like, 19. NADP-reducing hydrogenase subunit hndB, 20. nqoG, 21. CBS domain-containing protein, 22. Formate dehydrogenase Fe-S binding subunit, 23. Formate dehydrogenase subunit alpha, 24. LysM motif-containing metalloendopeptidase, 25 . coxL family molybdopterin aldehyde dehydrogenase. 


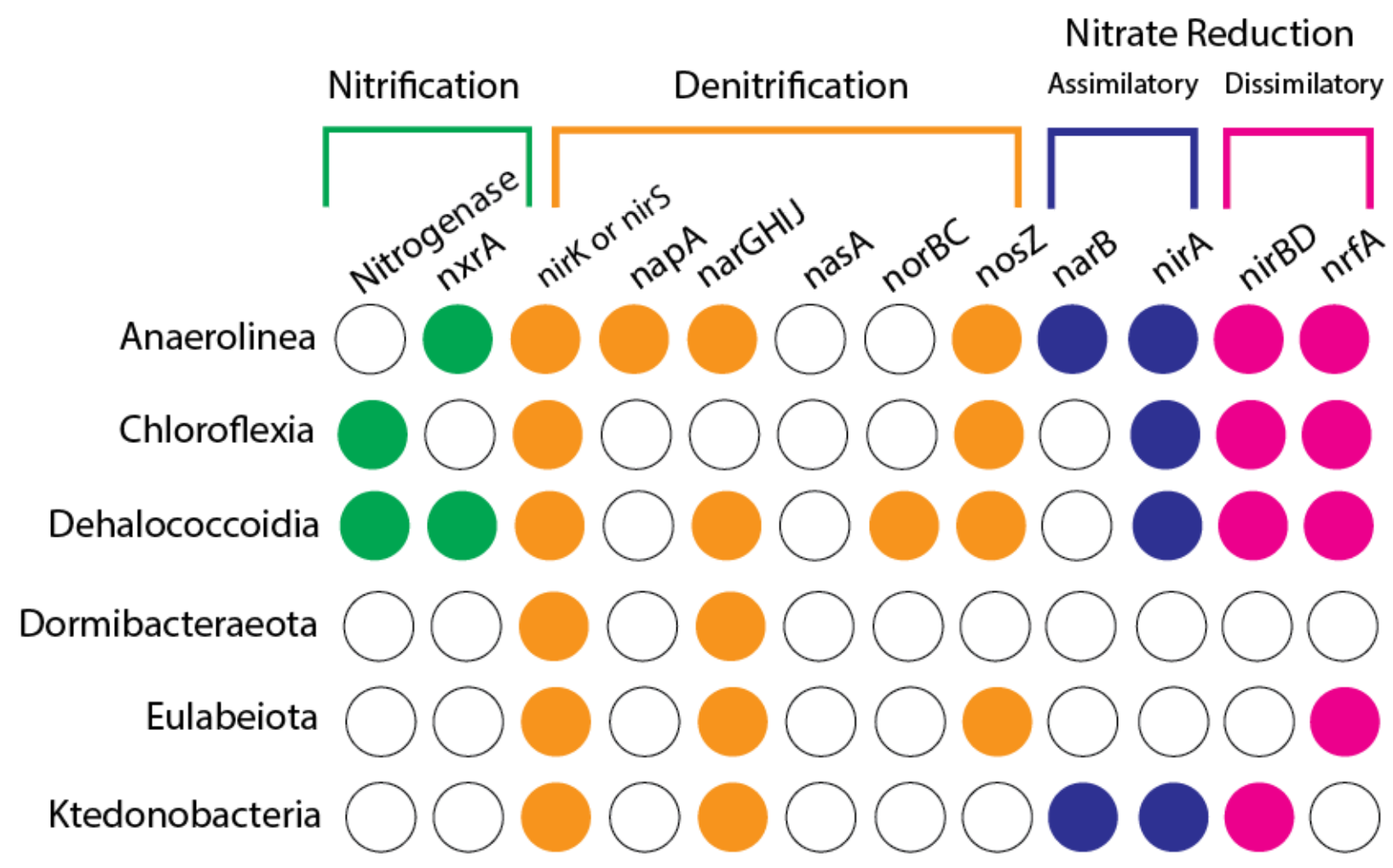

Figure 7. Colored circles indicate the presence or absence of important nitrogen cycling genes in major subdivisions of the Chloroflexi supergroup. 
bioRxiv preprint doi: https://doi.org/10.1101/2021.08.23.457424; this version posted August 24, 2021. The copyright holder for this preprint (which was not certified by peer review) is the author/funder, who has granted bioRxiv a license to display the preprint in perpetuity. It is made available under aCC-BY-ND 4.0 International license.

\section{Supplementary Figures}

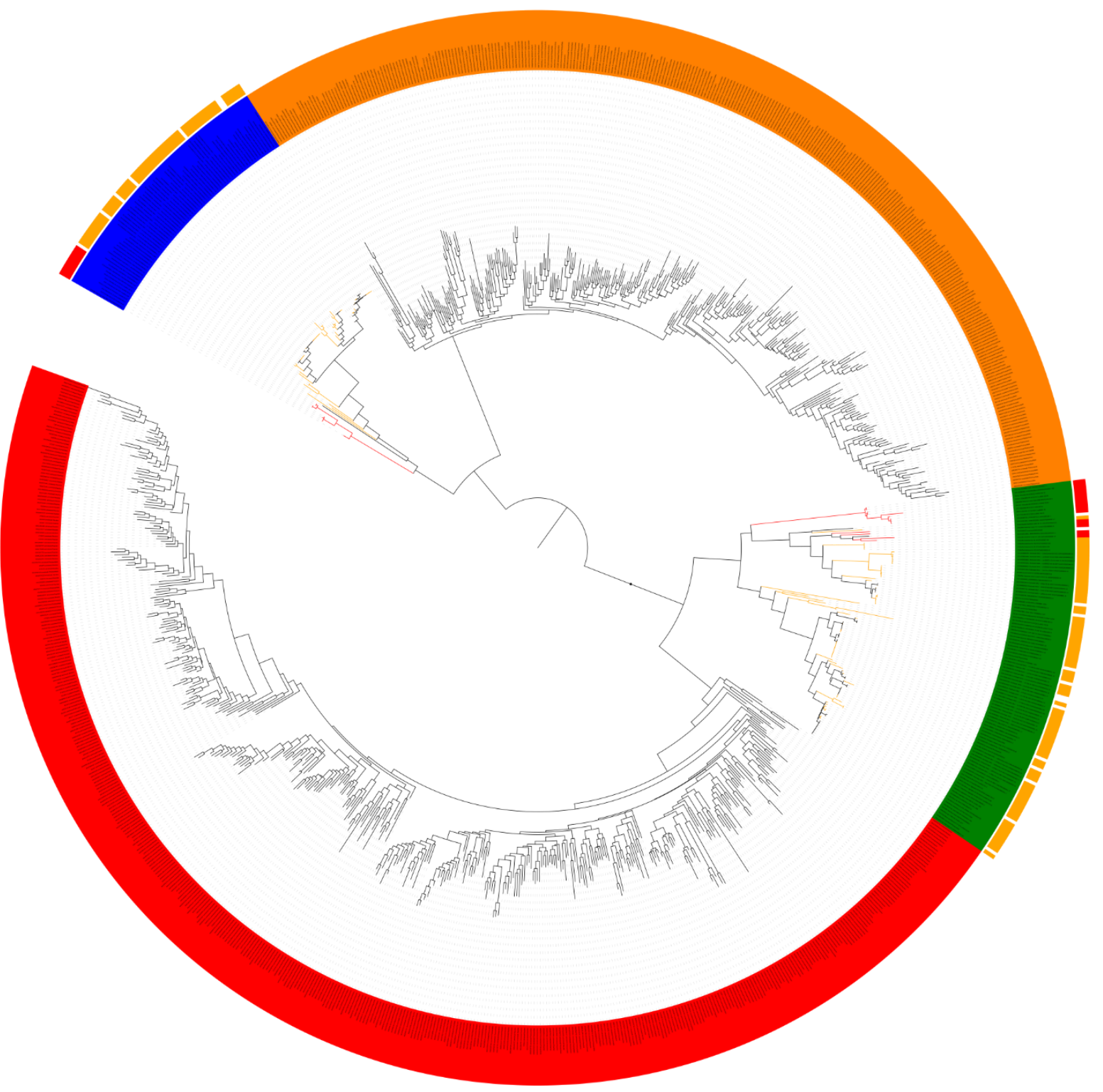

Tree scale: 1

\begin{tabular}{l} 
Colored ranges \\
\hline$\square$ pufM \\
$\square$ pufM Chloroflexi \\
pufL Chloroflexi \\
$\square$ pufL
\end{tabular}


Supplementary figure S1. Phylogeny of type II photosystem reaction centers pufLM with reference sequences from Uniprot. Sequences with decorations on the outer ring indicate pufLM sequences from the Chloroflexi supergroup genome dataset; red decorations indicate Anaerolinea and orange indicate Chloroflexia.

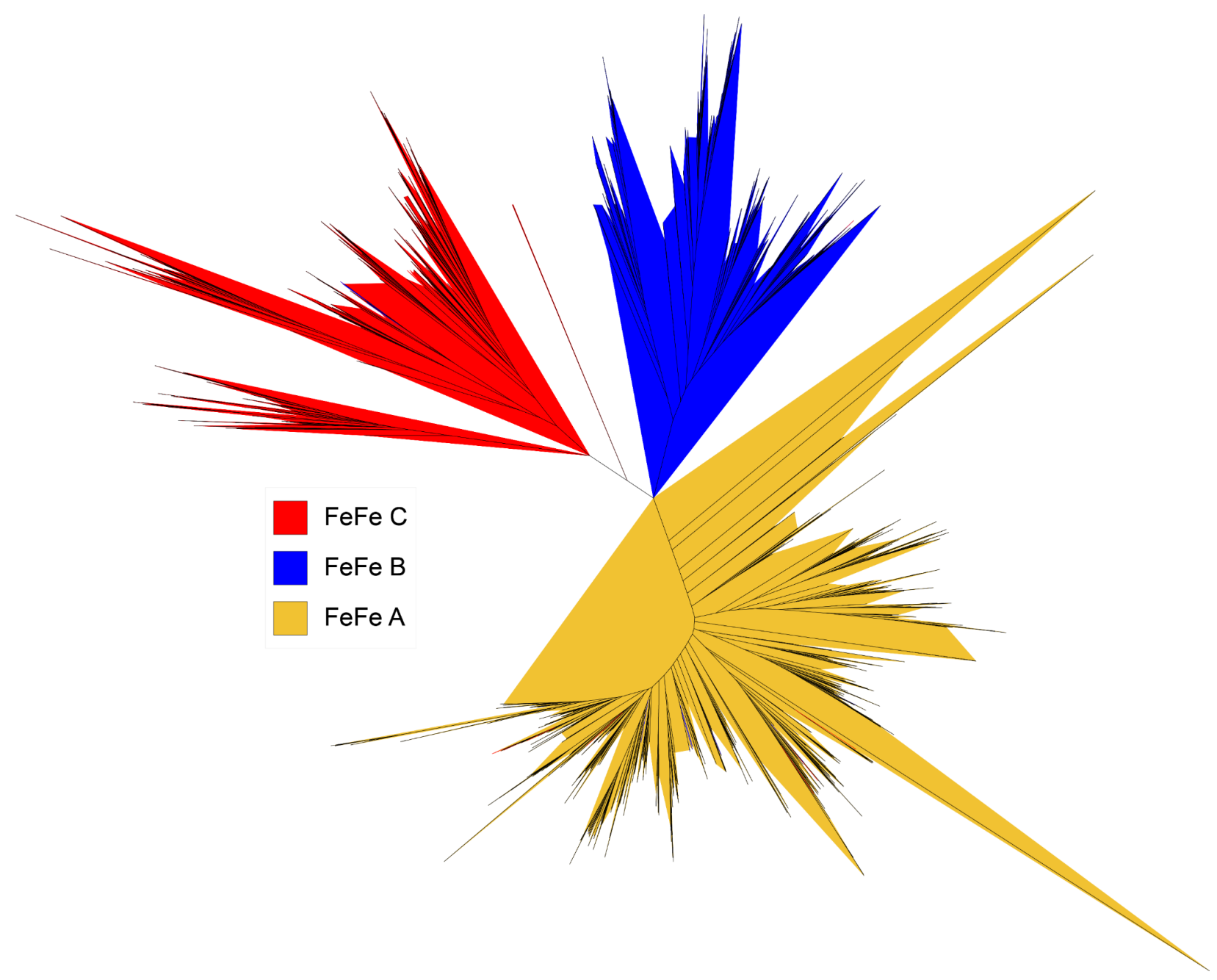

Supplementary figure S2. Phylogeny of FeFe hydrogenases in the Chloroflexi supergroup including references from HydDB and Matheus Carnevali et al. 2019. 


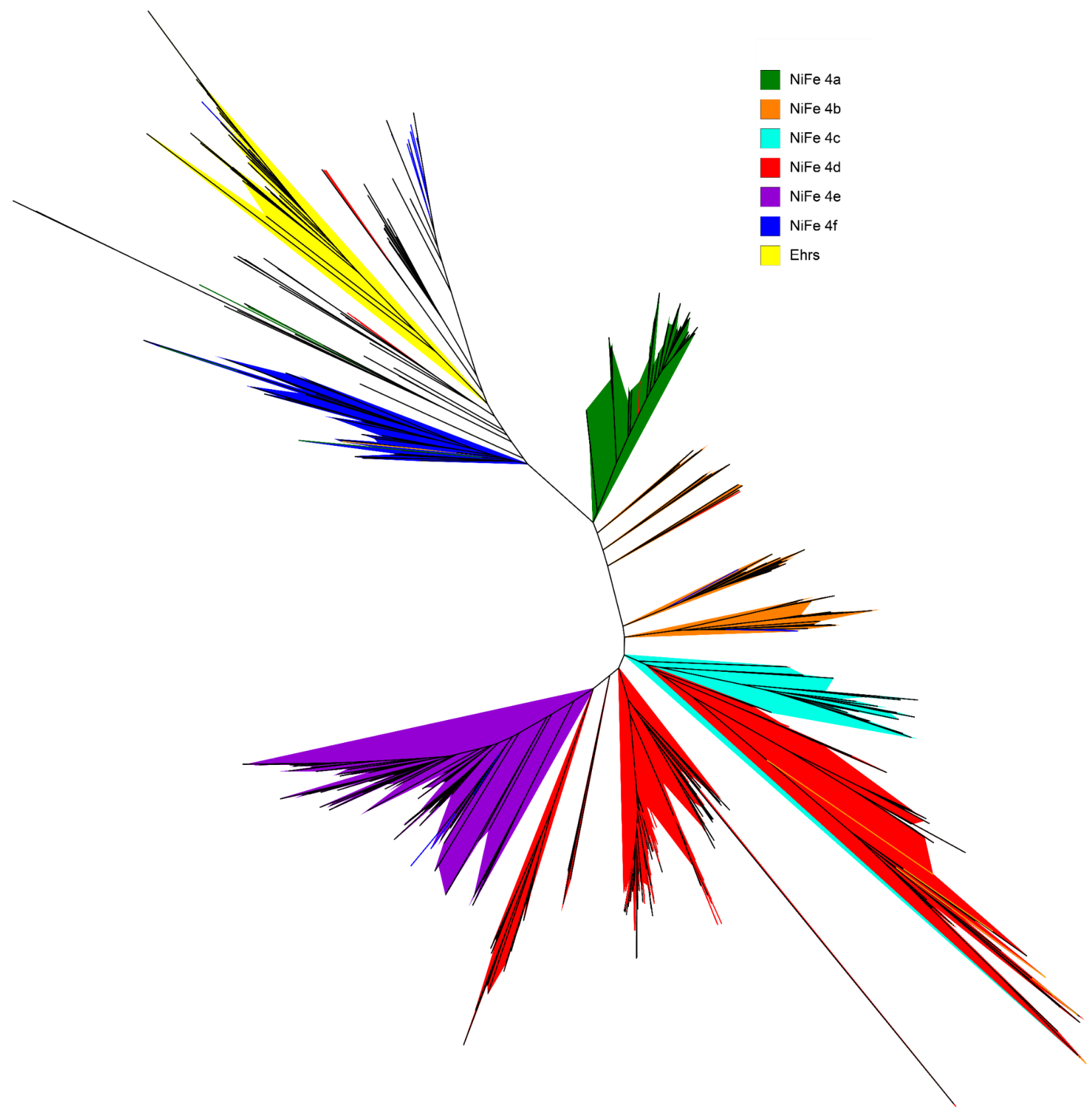

Supplementary figure S3. Phylogeny of NiFe group 4 hydrogenases in the Chloroflexi supergroup including references from HydDB and Matheus Carnevali et al. 2019. 


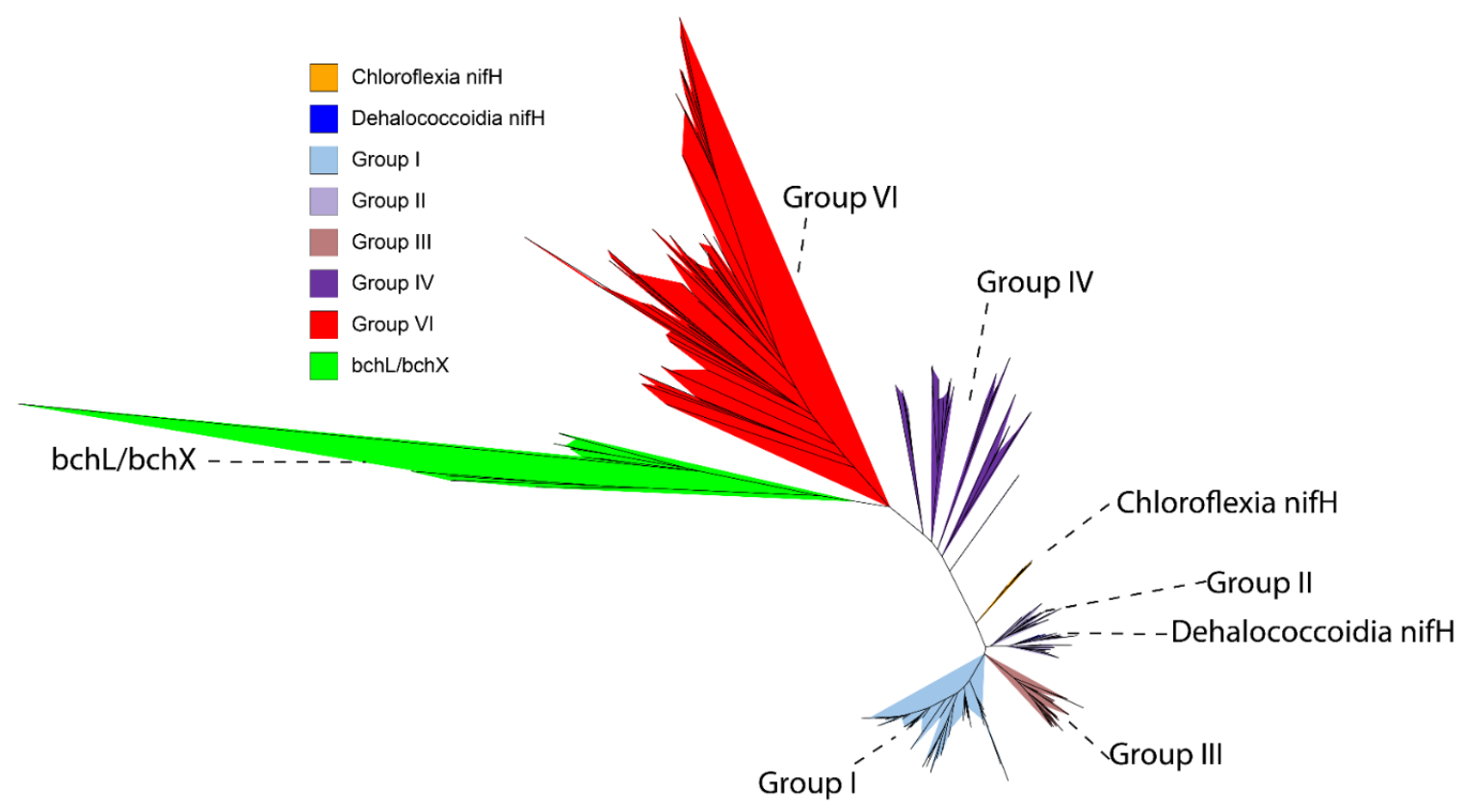

Supplementary figure S4. Phylogeny of nifH sequences with references from Uniprot and Meheust et al. 2020.

A

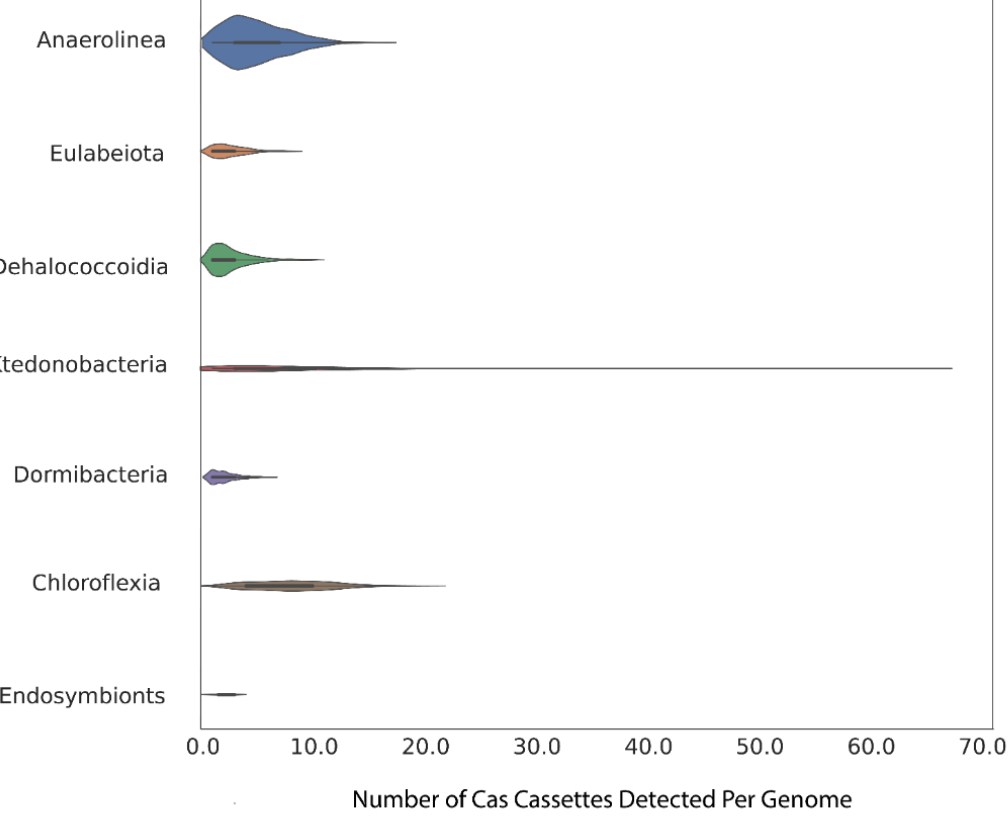

B

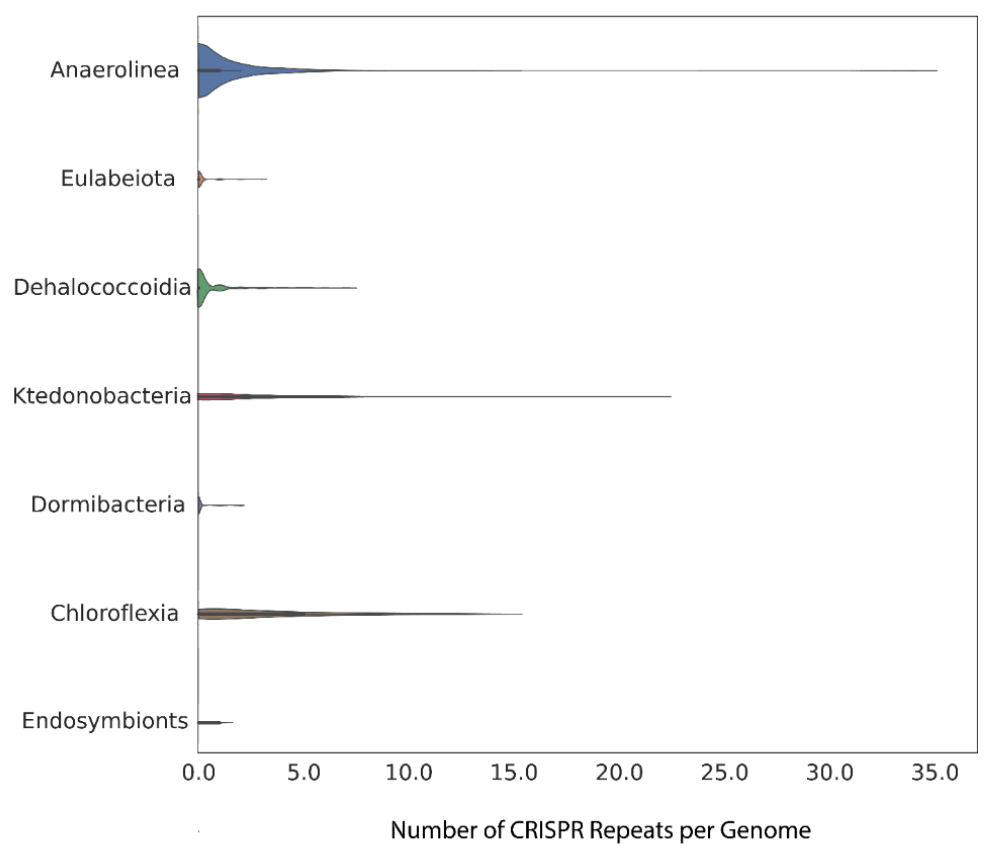

Supplementary figure S5. Violin plot of number of CRISPR loci by phyletic group within the Chloroflexi supergroup. 